MATHEMATICS OF COMPUTATION

Volume 72, Number 244, Pages 1611-1634

S 0025-5718(03)01507-2

Article electronically published on March 26, 2003

\title{
ON THE A POSTERIORI ERROR ANALYSIS FOR EQUATIONS OF PRESCRIBED MEAN CURVATURE
}

\author{
FRANCESCA FIERRO AND ANDREAS VEESER
}

\begin{abstract}
We present two approaches to the a posteriori error analysis for prescribed mean curvature equations. The main difference between them concerns the estimation of the residual: without or with computable weights. In the second case, the weights are related to the eigenvalues of the underlying operator and thus provide local and computable information about the conditioning. We analyze the two approaches from a theoretical viewpoint. Moreover, we investigate and compare the performance of the derived indicators in an adaptive procedure. Our theoretical and practical results show that it is advantageous to estimate the residual in a weighted way.
\end{abstract}

\section{INTRODUCTION}

Adaptive finite element methods are an effective tool for computing approximate solutions of partial differential equations. An important theoretical basis for these methods is a so-called a posteriori error analysis. In such analysis one tries to determine computable quantities that can be used to guide an adaptive procedure. For an overview of a posteriori error analyses and their applications, we refer to the seminal papers of Babuška and Rheinboldt [BR78a, BR78b, the books of Ainsworth and Oden [AO00] and Verfürth [Ver96], and the more recent developments of Dörfler [Dör96 and Morin et al. [MNS00, MNS].

This paper concerns the a posteriori error analysis and its application to the approximate solution of the following quasilinear, nonuniformly elliptic problem: find a function $u$ with boundary values $g$ and whose graph has mean curvature $H / d$, in formulae (see [GT83, (14.103)])

$$
-\operatorname{div}\left(\frac{\nabla u}{\sqrt{1+|\nabla u|^{2}}}\right)=H \quad \text { in } \Omega \subset \mathbb{R}^{d}, \quad u=g \quad \text { on } \partial \Omega .
$$

In order to survey our results concisely, we restrict ourselves for the rest of this introduction to the case of $d=2$, homogeneous boundary values $g=0$, and the existence of a classical solution to problem (1.1). Moreover, we focus on global upper bounds. In what follows, we discuss also local lower bounds, that is, how efficiently a certain kind of error is controlled. Let $u_{h}$ be the finite element approximation of

Received by the editor September 19, 2001 and, in revised form, March 27, 2002.

2000 Mathematics Subject Classification. Primary 65N30, 65N15; Secondary 35J25.

Key words and phrases. A posteriori error estimates, adaptive finite element methods, prescribed mean curvature equations, nonparametric minimal surfaces.

Research partially supported by the TMR network "Viscosity Solutions and Their Applications", the CNR Contract CU99.01713.CT01, and Italian M.I.U.R. Cofin2000 Project "Calcolo Scientifico: Modelli e Metodi Numerici Innovativi". 
$u$ over some triangulation $\mathcal{T}_{h}$ of $\Omega$. We shall use ' $\preccurlyeq$ ' to indicate inequalities that hold up to multiplicative constants depending solely on the minimum angle of $\mathcal{T}_{h}$.

We present and analyze two approaches to the a posteriori error analysis of problem (1.1). Both approaches yield an a posteriori control for a quantity $e_{\Omega}\left(u_{h}, u\right)$ which measures in a geometric way the distance between $\nabla u_{h}$ and $\nabla u$; see $\$ 3$ for details. Moreover, it has the property (recall that $g_{h}=g=0$ )

$$
e_{\Omega}\left(u_{h}, u\right)=0 \Longleftrightarrow u_{h}=u \text {. }
$$

The first approach follows the spirit of the general framework for elliptic problems proposed by Verfürth [Ver94] (see also [AO00, Ver96 and Fierro [Fie98): the analysis is built around the exact solution $u$ and measures the residual $\mathcal{R}_{h}$ of the discrete solution $u_{h}$ with respect to the equation in (1.1) in an unweighted dual norm. Triangle-indexed indicators $\eta_{h}(T), T \in \mathcal{T}_{h}$, with the following property are determined.

Result I. Let $M>0$ be fixed. If

$$
\sup _{\Omega}\left|\nabla\left(u_{h}-u\right)\right| \leq M,
$$

then

$$
e_{\Omega}\left(u_{h}, u\right) \preccurlyeq\left[\frac{\sum_{T \in \mathcal{T}_{h}} \eta_{h}(T)^{2}}{\inf _{\Omega} \lambda^{M}(\nabla u)}\right]^{1 / 2}
$$

with $\lambda^{M}(p):=\left[1+(|p|+M)^{2}\right]^{-3 / 2}$ for $p \in \mathbb{R}^{2}$.

Similarly to [Ver94, Proposition 6.1], this result has the following two drawbacks:

- Condition (1.2) cannot be verified in practice, because it involves the unknown exact solution $u$.

- In order to ensure with the help of (1.3) that $e_{\Omega}\left(u_{h}, u\right)$ is small, all indicators have to be small with respect to $\inf _{\Omega} \lambda^{M}(\nabla u)^{1 / 2}$. The latter quantity is small, iff $|\nabla u|$ is big somewhere. Besides, it is not computable. In other words: information on the conditioning, i.e., the relationship between residual (estimated by the indicators $\eta_{h}(T)$ ) and error, enters (1.3) in a global and noncomputable way.

The second approach tries to avoid these drawbacks. Its analysis is built around the computed solution $u_{h}$, and measures the residual $\mathcal{R}_{h}$ in weighted dual norms. We determine triangle-indexed weights $\lambda_{h}(T)$ and $Q_{h}(T), T \in \mathcal{T}_{h}$, such that the following result holds. We stress that both weigths $\lambda_{h}(T)$ and $Q_{h}(T)$ are computable and local expressions. Moreover, they are related to the eigenvalues of the elliptic operator in (1.1), and $\lambda_{h}(T), T \in \mathcal{T}_{h}$, is a local discrete counterpart of $\sup _{\Omega} \lambda^{0}(\nabla u)$; see $\$ 5.2$ for details. Denoting the diameter of a triangle $T \in \mathcal{T}_{h}$ by $h_{T}$, we have

Result II. There is a constant $C>0$, depending solely on the minimum angle of the triangulation $\mathcal{T}_{h}$, such that if

$$
\max _{T \in \mathcal{T}_{h}} Q_{h}(T) h_{T}^{-1} \eta_{h}(T) \leq C,
$$

then

$$
e_{\Omega}\left(u_{h}, u\right) \preccurlyeq\left[\sum_{T \in \mathcal{T}_{h}} \frac{\eta_{h}(T)^{2}}{\lambda_{h}(T)}\right]^{1 / 2} .
$$


In comparison with the discussion of Result I, we point out that:

- Given the computed solution $u_{h}$, condition (1.4) could be verified in principle. However, the realistic estimation of the constant $C$ is an open problem.

- The bound (1.5) appears to hold only conditionally, and condition (1.4) is related to closeness of $u_{h}$ to $u$. In particular, (1.2) with small $M>0$ implies (1.4) provided the prescribed mean curvature $H$ is resolved well. (See $\$ 5.4$ below.)

- To ensure with (1.5) that $e_{\Omega}\left(u_{h}, u\right)$ is small, each indicator $\eta_{h}(T), T \in$ $\mathcal{T}_{h}$, has to be small with respect to the computable $\lambda_{h}(T)^{1 / 2}$. The latter quantity is small, iff $\left|\nabla u_{h}\right|$ is big in a neighborhood of $T$. In other words: the conditioning enters in (1.5) in a local and computable way and can be incorporated in the indicators.

- If $\nabla u_{h} \approx \nabla u$ in $\Omega$, then (1.5) is sharper than (1.3) (apart from a multiplicative constant depending on the minimum angle of the triangulation $\left.\mathcal{T}_{h}\right)$.

Our numerical results complement the above discussion by indicating that:

- Condition (1.4) is "asymptotically satisfied", and the constants hidden in "ß" are of moderate size on meshes with reasonable minumum angle.

- Weighted indicators (see below) direct the adaptive procedure more efficiently than unweighted ones.

The rest of this article is organized as follows. In $\$ 2$ we state a weak formulation of problem (1.1) and its finite element discretization. Moreover, we give a condition that characterizes the unique solvability of the discrete problem. Then, in 93 , we introduce the error $e_{\Omega}\left(u_{h}, u\right)$, the residual $\mathcal{R}_{h}$ of $u_{h}$, and give some interpolation error estimates. In $\S \$ 45$ we present and analyze the two approaches illustrated by Results I and II. In $\sqrt{6}$ we discuss the application of both approaches in an adaptive algorithm. Furthermore, we comment on our implementation. Finally, we investigate our theoretical results from the practical viewpoint in 47 .

\section{Continuous PRoblem AND its DisCRETIZATion}

We begin by stating a weak form of the Dirichlet problem for the prescribed mean curvature equation. Taking advantage of its divergence structure, we then discretize with continuous linear finite elements.

Let $\Omega$ be a bounded domain with Lipschitz boundary in $\mathbb{R}^{d}, d \geq 2$. For $k \in \mathbb{N}_{0}$ and $q \in[1, \infty], W_{q}^{k}(\Omega)$ denotes the Banach space of functions whose distributional derivatives up to order $k$ are Lebesgue-measurable and $q$-integrable functions. The norm in $L_{q}(\Omega)=W_{q}^{0}(\Omega)$ is indicated by $\|\cdot\|_{0, q ; \Omega}$. If $q=2$, we write also $H^{k}(\Omega)$ for $W_{2}^{k}(\Omega)$. Analogous notation is used for fractional order Sobolev spaces on $\partial \Omega$.

It is convenient to introduce the functions $A: \mathbb{R}^{d} \rightarrow \mathbb{R}$ and $a: \mathbb{R}^{d} \rightarrow \mathbb{R}^{d}$ :

$$
A(p):=\sqrt{1+|p|^{2}} \text { and } a(p):=\nabla A(p)=\frac{p}{\sqrt{1+|p|^{2}}} .
$$

Let $g \in H^{1 / 2}(\partial \Omega)=\left\{\hat{g}_{\mid \partial \Omega} \mid \hat{g} \in H^{1}(\Omega)\right\}$, see e.g., [Hac92 Section 6.2.5], and $H \in L_{2}(\Omega)$. In the sequel, we ignore the factor $1 / d$ and call $H$ the prescribed mean curvature. We define the space of test functions as $V:=\left\{w \in H^{1}(\Omega) \mid w_{\mid \partial \Omega}=0\right\}$ and associate with $g$ the affine space $U:=\left\{w \in H^{1}(\Omega) \mid w_{\mid \partial \Omega}=g\right\}$. The following problem is a weak form of (1.1), which is convenient for the following sections. 
Problem 2.1 (Weak form). Find $u \in U$ such that

$$
\forall v \in V \quad \int_{\Omega} a(\nabla u) \cdot \nabla v=\int_{\Omega} H v
$$

We proceed by stating some well-known facts about the classification and the solvability of Problem 2.1 ,

Let $\lambda(p)$ and $\Lambda(p)$ be respectively the minimum and maximum eigenvalue of the Hessian $D^{2} A(p), p \in \mathbb{R}^{d}$. Since

$$
\begin{gathered}
\lambda(p)=\left(1+|p|^{2}\right)^{-3 / 2}>0, \quad \Lambda(p)=\left(1+|p|^{2}\right)^{-1 / 2}, \\
Q(p):=\frac{\Lambda(p)}{\lambda(p)}=1+|p|^{2} \rightarrow \infty \quad \text { as } \quad|p| \rightarrow \infty,
\end{gathered}
$$

the operator associated with equation (2.1) is nonuniformly elliptic. Moreover, it is quasilinear and variational (e.g., see the proof of Proposition 2.1 below).

Problem 2.1 has at most one solution (see e.g., Proposition 3.1 below). However, its solvability is a delicate matter. For example, it may not be solvable classically for smooth data; see Serrin [Ser69]. Here, we are interested in solvability in $W_{\infty}^{1}(\Omega)$. A necessary condition on the prescribed mean curvature $H$ for that is

$$
\exists \epsilon_{0}>0 \quad \forall v \in V \quad \int_{\Omega} H v \leq\left(1-\epsilon_{0}\right) \int_{\Omega}|\nabla v| .
$$

This follows from (2.1) and $|a(\nabla u)| \leq\|\nabla u\|_{0, \infty ; \Omega} /\left(1+\|\nabla u\|_{0, \infty ; \Omega}^{2}\right)^{1 / 2}<1$ for $u$ in $W_{\infty}^{1}(\Omega)$. Sufficient conditions on data for the solvability in $W_{\infty}^{1}(\Omega)$ (and better) can be found in Giaquinta [Gia74] and Gilbarg and Trudinger [GT83, Theorem 16.10].

For the discretization, suppose that the domain $\Omega$ is polyhedral and let $\mathcal{T}_{h}$ be a conforming triangulation of $\Omega$. Let $\widehat{T}$ denote the standard simplex in $\mathbb{R}^{d}$. Given a simplex $T \in \mathcal{T}_{h}$, we define $T^{*}$ as the union of all simplices in $\mathcal{T}_{h}$ that share at least one side with $T$, and $B_{h}(T)$ as the union of all simplices in $\mathcal{T}_{h}$ that have nonempty intersection with $T$. Moreover, we write $\rho_{T}$ for the maximal radius of a ball that is contained in $T$, and $h_{T}$ for the diameter of $T$. The shape-regularity of the triangulation $\mathcal{T}_{h}$ is defined by

$$
\left.\gamma_{h}:=\max _{T \in \mathcal{T}_{h}} \frac{h_{T}}{\rho_{T}} \in\right] 1, \infty[.
$$

In dimension two it is related to the minimum angle of $\mathcal{T}_{h}$; see e.g., Cia78, Exercise 3.1.3]. In the sequel, the same letter $C$ will be used to denote different constants depending on the shape-regularity $\gamma_{h}$. For example, the number of simplices in $B_{h}(T)$ for any $T \in \mathcal{T}_{h}$ is bounded by a constant depending on $\gamma_{h}$,

$$
\#\left\{\widetilde{T} \in \mathcal{T}_{h} \mid \widetilde{T} \subset B_{h}(T)\right\} \leq C .
$$

We will also write $\preccurlyeq$ instead of $\leq C$; e.g., $\left|B_{h}(T)\right| \preccurlyeq h_{T}^{d}$. Let $\mathcal{N}_{h}$ be the set of nodes and $\mathcal{S}_{h}$ the set of sides of $\mathcal{T}_{h}$. Both sets $\mathcal{N}_{h}$ and $\mathcal{S}_{h}$ consist of two parts: the "boundary" parts $\mathcal{N}_{h}^{\partial}:=\mathcal{N}_{h} \cap \partial \Omega$ and $\mathcal{S}_{h}^{\partial}:=\left\{S \in \mathcal{S}_{h} \mid S \subset \partial \Omega\right\}$ as well as the "interior" parts $\mathcal{N}_{h}^{\circ}:=\mathcal{N}_{h} \cap \Omega$ and $\mathcal{S}_{h}^{\circ}:=\mathcal{S}_{h} \backslash \mathcal{S}_{h}^{\partial}$. Furthermore, we use the local mesh size function $h \in L_{\infty}(\Omega)$ defined by $h_{\mid T}=h_{T}$ for all $T \in \mathcal{T}_{h}$.

Let $W_{h}$ denote the finite-dimensional space of continuous piecewise affine finite elements over $\mathcal{T}_{h}$; that is,

$$
W_{h}:=\left\{w_{h} \in C^{0}(\bar{\Omega}) \mid \forall T \in \mathcal{T}_{h} w_{h \mid T} \in P_{1}(T)\right\} \subset H^{1}(\Omega) .
$$


A POSTERIORI ANALYSIS FOR PRESCRIBED MEAN CURVATURE EQUATIONS 1615

Hereafter, $P_{m}(T)$ denotes the restriction to $T$ of the space of all polynomials in $\mathbb{R}^{d}$ with degree equal to or less than $m \in \mathbb{N}_{0}$. Let $\left(\phi_{h}^{z}\right)_{z \in \mathcal{N}_{h}}$ be the hat functions that are defined by $\phi_{h}^{z} \in W_{h}, \phi_{h}^{z}(z)=1$ and $\phi_{h}^{z}(\bar{z})=0$ for all $\bar{z} \in \mathcal{N}_{h} \backslash\{z\}$. Moreover, set

$$
V_{h}:=\left\{w_{h} \in W_{h} \mid w_{h}=0 \text { on } \partial \Omega\right\} \subset V, \quad U_{h}:=\left\{w_{h} \in W_{h} \mid w_{h}=g_{h} \text { on } \partial \Omega\right\},
$$

where $g_{h} \in W_{h \mid \partial \Omega}:=\left\{\hat{g}_{h \mid \partial \Omega} \mid \hat{g}_{h} \in W_{h}\right\}$ is an approximation of the boundary values $g$. Note that $U_{h}$ is contained in $U$, iff $g=g_{h}$.

We approximate solutions of Problem 2.1 by means of solutions of the following finite-dimensional problem.

Problem 2.2 (Discrete problem). Find $u_{h} \in U_{h}$ such that

$$
\forall v_{h} \in V_{h} \quad \int_{\Omega} a\left(\nabla u_{h}\right) \cdot \nabla v_{h}=\int_{\Omega} H v_{h} .
$$

Problem 2.2 has a unique solution, if Problem 2.1 has one in $W_{\infty}^{1}(\Omega)$. More precisely, we have the following statement.

Proposition 2.1 (Discrete solvability). Problem 2.2 is uniquely solvable, iff the discrete counterpart of condition (2.2) holds:

$$
\exists \epsilon_{0}>0 \quad \forall v_{h} \in V_{h} \quad \int_{\Omega} H v_{h} \leq\left(1-\epsilon_{0}\right) \int_{\Omega}\left|\nabla v_{h}\right|
$$

Proof. Since $V_{h} \subset W_{\infty}^{1}(\Omega)$, the necessity of condition 2.5 is clear. For its sufficiency, we first observe that Problem 2.2 is equivalent to the minimization of the convex and continuous functional

$$
J\left(w_{h}\right):=\int_{\Omega} A\left(\nabla w_{h}\right)-H w_{h}
$$

in $U_{h}$; see [Cia78, Section 5.2], where the case $H=0$ is considered. It therefore suffices to establish the unique existence of such a minimum. Let $w_{h} \in U_{h}$, and let $\hat{g}_{h} \in W_{h}$ be an extension of $g_{h}$. Using condition (2.5) with $v_{h}=w_{h}-\hat{g}_{h} \in V_{h}$, we find that

$$
J\left(w_{h}\right) \geq \epsilon_{0}\left\|\nabla w_{h}\right\|_{0,1 ; \Omega}-\left(\left\|\nabla \hat{g}_{h}\right\|_{0,1 ; \Omega}+\int_{\Omega} H \hat{g}_{h}\right) .
$$

The functional $J$ is thus coercive and has a minimum; see e.g., Theorem $8.2-1$ in Cia88.

The uniqueness of the minimum follows from the strict convexity of $A$ or from Proposition 3.1 below.

\section{ERROR, RESIDUAL, AND INTERPOLATION}

We introduce and motivate the error notion. Moreover, we introduce a fundamental quantity for the error control, the residual, and state some error estimates for interpolation of nonsmooth functions.

3.1. The error. We begin with an identity concerning differences of the principal terms in (2.1) and (2.4).

Lemma 3.1. Let $p_{1}, p_{2} \in \mathbb{R}^{d}$. Defining $P_{i}:=\left(p_{i},-1\right) \in \mathbb{R}^{d+1}$ for $i=1,2$, we have

$$
\left[a\left(p_{1}\right)-a\left(p_{2}\right)\right] \cdot\left(p_{1}-p_{2}\right)=\left|\frac{P_{1}}{\left|P_{1}\right|}-\frac{P_{2}}{\left|P_{2}\right|}\right|^{2} \frac{\left|P_{1}\right|+\left|P_{2}\right|}{2} .
$$


Proof. We calculate (note that all dots after the first equal sign denote the scalar product in $\mathbb{R}^{d+1}$ )

$$
\begin{aligned}
{\left[a\left(p_{1}\right)-a\left(p_{2}\right)\right] } & \cdot\left(p_{1}-p_{2}\right)=\left(\frac{P_{1}}{\left|P_{1}\right|}-\frac{P_{2}}{\left|P_{2}\right|}\right) \cdot\left(P_{1}-P_{2}\right) \\
& =\left(\frac{P_{1}}{\left|P_{1}\right|}-\frac{P_{2}}{\left|P_{2}\right|}\right) \cdot \frac{P_{1}}{\left|P_{1}\right|}\left|P_{1}\right|+\left(\frac{P_{2}}{\left|P_{2}\right|}-\frac{P_{1}}{\left|P_{1}\right|}\right) \cdot \frac{P_{2}}{\left|P_{2}\right|}\left|P_{2}\right| .
\end{aligned}
$$

Since $\left(N_{1}-N_{2}\right) \cdot N_{1}=\frac{1}{2}\left|N_{1}-N_{2}\right|^{2}$ for $\left|N_{1}\right|=\left|N_{2}\right|=1$, we arrive at (3.1).

In view of the geometric meaning of problem (1.1), it is worthwhile recognizing the following interpretation of the right-hand side in (3.1): for $p_{1}=\nabla u(x), x \in \Omega$, we have that $\left|P_{1}\right|$ is the area element and $P_{1} /\left|P_{1}\right|$ is the downwards pointing normal of $u$ 's graph in $(x, u(x))$. By this and the weak equations (2.1) and (2.4), we are led to the following definition.

Definition 3.1 (Error notion). Let $\omega$ be a subdomain of $\Omega$ and $u, v \in W_{1}^{1}(\Omega)$. Set

$$
e_{\omega}(u, v):=\left(\int_{\omega}|N(\nabla u)-N(\nabla v)|^{2} \frac{A(\nabla u)+A(\nabla v)}{2}\right)^{1 / 2},
$$

where $N(p):=(p,-1) / \sqrt{1+|p|^{2}} \in \mathbb{R}^{d+1}$ for $p \in \mathbb{R}^{d}$.

The quantity $e_{\Omega}\left(u_{h}, u\right)$, where $u_{h}$ and $u$ are the solutions of Problems 2.2 and 2.1] respectively, will be the error that is bounded from above and locally from below in the subsequent analyses. The geometric interpretation preceding Definition 3.1 yields the following: $e_{\Omega}\left(u_{h}, u\right)$ measures the distance between the normals $N\left(\nabla u_{h}\right)$ and $N(\nabla u)$ in a weighted $L_{2}$-sense, where the weight is the average of the area elements of the graphs of $u_{h}$ and $u$. The control of $e_{\Omega}\left(u_{h}, u\right)$ is therefore interesting in its own right. Moreover, as can be seen from the following remark, $e_{\Omega}\left(u_{h}, u\right)$ is closely related to the approximation error of the minimum value of $J$ in the set $U$. This value is especially interesting in applications, where the prescribed mean curvature vanishes, i.e., $H=0$. The minimum value of $J$ is then the minimal area of the graphs with boundary values $g$.

Remark 3.1 (Error estimate for minimum of $J$ ). Suppose that we want to approximate the value $J(u)$, where $u$ is a solution of Problem 2.1. Given a solution $u_{h}$ of Problem 2.2 a natural choice for the approximation of $J(u)$ is $J\left(u_{h}\right)$. Consequently, we want to control the error $J\left(u_{h}\right)-J(u)$. For the sake of simplicity, let us suppose that $g=g_{h}$. Then $U_{h} \subseteq U$, and we do not have to take consistency errors into account. Thus, on the one hand, $J\left(u_{h}\right)-J(u) \geq 0$. On the other hand, we have

$$
\begin{aligned}
J(u)-J\left(u_{h}\right) & \geq \int_{\Omega} a\left(\nabla u_{h}\right) \cdot \nabla\left(u-u_{h}\right)-H\left(u-u_{h}\right) \\
& =\int_{\Omega}\left[a\left(\nabla u_{h}\right)-a(\nabla u)\right] \cdot \nabla\left(u-u_{h}\right)=-e_{\Omega}\left(u_{h}, u\right)^{2}
\end{aligned}
$$

by the convexity of $A$ (cf. Cia88, Theorem 7.4-2]), $a=\nabla A$, the weak equation (2.1), $U_{h} \subseteq U$, and Lemma 3.1 Combining the two inequalities yields

$$
0 \leq J\left(u_{h}\right)-J(u) \leq e_{\Omega}\left(u_{h}, u\right)^{2} .
$$

We point out that $e_{\Omega}\left(u_{h}, u\right)$ is more directly related to $J\left(u_{h}\right)-J(u)$ than, for example, the norm $\left\|\nabla\left(u_{h}-u\right)\right\|_{0,2 ; \Omega}$; see also (4.11) below. 
We conclude this subsection with a uniqueness proof for solutions of Problem 2.1] (or Problem 2.2) which sheds some light on the ellipticity properties of $e_{\Omega}$.

Proposition 3.1 (Uniqueness). Problem 2.1 has at most one solution. The same holds for Problem 2.2.

Proof. We verify only the uniqueness of Problem 2.1 Problem 2.2 goes along the same lines. Let $u_{1}$ and $u_{2}$ be two solutions of Problem 2.1. Testing the equations for $u_{1}$ and $u_{2}$ with $v=u_{1}-u_{2} \in V$ yields $e_{\Omega}\left(u_{1}, u_{2}\right)=0$, and thus

$$
N\left(\nabla u_{1}\right)=N\left(\nabla u_{2}\right) \quad \text { a.e. in } \Omega \text {. }
$$

The last coordinate of (3.2) ensures $A\left(\nabla u_{1}\right)=A\left(\nabla u_{2}\right)$ in $\Omega$. Consequently, the first $d$ coordinates yield $\nabla u_{1}=\nabla u_{2}$ in $\Omega$. Since $u_{1}-u_{2} \in V$, we get $u_{1}=u_{2}$.

3.2. The residual. The following definition will provide a link between the error and the computable quantities estimating the error.

As before, $u$ denotes the solution of Problem 2.1 and $u_{h}$ is the corresponding discrete solution of Problem 2.2. The residual $\mathcal{R}_{h} \in V^{*}$ of $u_{h}$ is defined by

$$
\forall \varphi \in V \quad\left\langle\mathcal{R}_{h}, \varphi\right\rangle:=\int_{\Omega} a\left(\nabla u_{h}\right) \cdot \nabla \varphi-\int_{\Omega} H \varphi
$$

The equation (2.4) for $u_{h}$ means

$$
\forall \varphi_{h} \in V_{h} \quad\left\langle\mathcal{R}_{h}, \varphi_{h}\right\rangle=0 .
$$

On the one hand, we have

$$
\forall \varphi \in V \quad\left\langle\mathcal{R}_{h}, \varphi\right\rangle=\int_{\Omega}\left[a\left(\nabla u_{h}\right)-a(\nabla u)\right] \cdot \nabla \varphi
$$

thanks to (2.1), and, if $g_{h}=g$ and therefore $U_{h} \subset U$,

$$
\left\langle\mathcal{R}_{h}, u_{h}-u\right\rangle=e_{\Omega}\left(u_{h}, u\right)^{2} .
$$

On the other hand, for any $\varphi \in V$, integrating by parts on each simplex yields

$$
\left\langle\mathcal{R}_{h}, \varphi\right\rangle=-\sum_{T \in \mathcal{T}_{h}}\left[\frac{1}{2} \int_{\partial T \backslash \partial \Omega} \llbracket a\left(\nabla u_{h}\right) \cdot n \rrbracket \varphi+\int_{T} H \varphi\right],
$$

where $\llbracket a\left(\nabla u_{h}\right) \cdot n \rrbracket$ denotes the function on $\bigcup_{S \in \mathcal{S}_{h}^{\circ}} S$ defined as follows: for any interelement side $S \in \mathcal{S}_{h}^{\circ}$ let $\left.\llbracket a\left(\nabla u_{h}\right) \cdot n \rrbracket\right|_{S}=\left[a\left(\nabla u_{h \mid T_{1}}\right)-a\left(\nabla u_{h \mid T_{2}}\right)\right] \cdot n_{2}$, where $T_{1}, T_{2} \in \mathcal{T}_{h}$ are the two simplices containing $S, n_{2}$ is the exterior normal of $T_{2}$, and the right-hand side is invariant if $T_{1}$ and $T_{2}$ are exchanged.

3.3. Interpolation error estimates. Let $\Pi_{h}: W_{1}^{1}(\Omega) \rightarrow W_{h}$ be the interpolation operator in Scott and Zhang [SZ90], which preserves homogeneous boundary values:

$$
\forall \varphi \in W_{1}^{1}(\Omega) \quad \varphi=0 \text { on } \partial \Omega \Longrightarrow \Pi_{h} \varphi=0 \text { on } \partial \Omega .
$$

The following local interpolation error estimates will be useful: for any $r, q \in[1, \infty[$, $T \in \mathcal{T}_{h}$, and $S \in \mathcal{S}_{h}$,

$$
\begin{aligned}
& \left\|\Pi_{h} \varphi-\varphi\right\|_{0, r ; T} \preccurlyeq h_{T}^{1-d / q+d / r}\|\nabla \varphi\|_{0, q ; B_{h}(T)}, \quad \text { if }-d / r \leq 1-d / q, \\
& \left\|\Pi_{h} \varphi-\varphi\right\|_{0, q ; S} \preccurlyeq h^{1-1 / q}\|\nabla \varphi\|_{0, q ; B_{h}(T)}, \quad \text { if } S \subset T \text {. }
\end{aligned}
$$


For convenience of the reader, we give a proof of (3.8a that is a slight modification of the proof for [SZ90, (4.3)], which covers the special case $r=q$. Using $\Pi_{h} c=c$ for any constant function $c$ [SZ90, (2.18)], we start with

$$
\left\|\Pi_{h} \varphi-\varphi\right\|_{0, r ; T} \leq\|\varphi-c\|_{0, r ; T}+\left\|\Pi_{h}(\varphi-c)\right\|_{0, r ; T} .
$$

The first term on the right-hand side is estimated by using scaling arguments (see e.g., [Cia78 Th. 3.1.2 and 3.1.3]) and, exploiting $-d / r \leq 1-d / q$, the Sobolev imbedding $W_{q}^{1}(\widehat{T}) \hookrightarrow L_{r}(\widehat{T})$ (see e.g., [Cia78, (3.1.3)]) over the reference simplex $\widehat{T}:$

$$
\|\varphi-c\|_{0, r ; T} \preccurlyeq h_{T}^{-d / q+d / r}\|\varphi-c\|_{0, q ; T}+h_{T}^{1-d / q+d / r}\|\nabla \varphi\|_{0, q ; T} .
$$

For the second one, the stability of $\Pi_{h}$, [SZ90, Theorem 3.1], yields similar terms:

$$
\left\|\Pi_{h}(\varphi-c)\right\|_{0, r ; T} \preccurlyeq h_{T}^{-d / q+d / r}\|\varphi-c\|_{0, q ; B_{h}(T)}+h_{T}^{1-d / q+d / r}\|\nabla \varphi\|_{0, q ; B_{h}(T)} .
$$

We obtain (3.8a) with the help of the following variant of the Bramble-Hilbert lemma, see [SZ90, (4.2)]:

$$
\inf _{c \in \mathbb{R}}\|\varphi-c\|_{0, q ; B_{h}(T)} \preccurlyeq h_{T}\|\nabla \varphi\|_{0, q ; B_{h}(T)} .
$$

Inequality (3.8b) follows from the "scaled" trace theorem

$$
\|\varphi\|_{0, q ; S} \preccurlyeq h_{T}^{-1 / q}\|\varphi\|_{0, q ; T}+h_{T}^{1-1 / q}\|\nabla \varphi\|_{0, q ; T} \quad \text { for } S \subset T
$$

(use scaling arguments and e.g., Hac92, Theorems 6.2.40 and 6.2.25]), (3.8a) with $r=q$, and a local estimate for the gradient of the interpolation error [SZ90, (4.3)].

\section{Approach I}

We present and analyze the first approach, which was illustrated by Result I in the introduction $\mathbb{1}$.

We start by introducing some notation. For any simplex $T \in \mathcal{T}_{h}$, we define the local indicator $\eta_{h}(T) \geq 0$ by

$$
\eta_{h}(T)^{2}=h_{T}\left\|\llbracket a\left(\nabla u_{h}\right) \cdot n \rrbracket\right\|_{0,2 ; \partial T \backslash \partial \Omega}^{2}+h_{T}^{2}\|H\|_{0,2 ; T}^{2} .
$$

Apart from the indicators, we shall use the $L_{2}$-oscillation of the prescribed mean curvature $H$ in a subdomain $\omega$ of $\Omega$. This is the quantity $\|h(\bar{H}-H)\|_{0,2 ; \omega}$, where $\bar{H} \in L_{\infty}(\Omega)$ is the piecewise constant function such that $\bar{H}_{\mid T}=|T|^{-1} \int_{T} H$ for all simplices $T \in \mathcal{T}_{h}$. The following theorem relies on measuring the residual $\mathcal{R}_{h}$ by

$$
\left\|\mathcal{R}_{h}\right\|_{-1,2 ; \omega}:=\sup \left\{\left\langle\mathcal{R}_{h}, \varphi\right\rangle \mid \varphi \in V, \operatorname{supp} \varphi \subseteq \bar{\omega},\|\nabla \varphi\|_{0,2 ; \omega} \leq 1\right\}
$$

in a nonempty subdomain $\omega$ of $\Omega$.

Theorem 4.1 (Approach I). Let $u$ be a solution of Problem [2.1] with $u \in W_{\infty}^{1}(\Omega)$, and let $u_{h}$ be the solution of Problem 2.2. Then

(i) (Local lower bounds) for any simplex $T \in \mathcal{T}_{h}$, we have

$$
\frac{\eta_{h}(T)}{\sup _{T^{*}} \Lambda(\nabla u)^{1 / 2}} \preccurlyeq e_{T^{*}}\left(u_{h}, u\right)+\frac{\|h(\bar{H}-H)\|_{0,2 ; T^{*}}}{\sup _{T^{*}} \Lambda(\nabla u)^{1 / 2}},
$$


(ii) (Conditional global upper bound) if

$$
\left\|\nabla\left(u_{h}-u\right)\right\|_{0, \infty ; \Omega} \leq M
$$

for some fixed $M>0$, then

$e_{\Omega}\left(u_{h}, u\right) \preccurlyeq\left(\frac{\sum_{T \in \mathcal{T}_{h}} \eta_{h}(T)^{2}}{\inf _{\Omega} \lambda^{M}(\nabla u)}+\sup _{\Omega} \Lambda(\nabla u)\left\|\nabla\left(\hat{g}_{h}-\hat{g}\right)\right\|_{0,2 ; \Omega}^{2}\right)^{1 / 2}$.

Here, $\lambda^{M}(p):=\left[1+(|p|+M)^{2}\right]^{-3 / 2}$, and $\hat{g}_{h}$, and $\hat{g}$ are $H^{1}(\Omega)$-extensions of $g_{h}$ and $g$.

Remark 4.1 (On the choice of $\hat{g}$ and $\hat{g}_{h}$ ). One may choose $\hat{g}$ and $\hat{g}_{h}$ as the harmonic extensions of $g$ and $g_{h}$. Then $\left\|\nabla\left(\hat{g}_{h}-\hat{g}\right)\right\|_{0,2 ; \Omega} \leq C_{\Omega}\left\|\hat{g}_{h}-\hat{g}\right\|_{H^{1 / 2}(\partial \Omega)}$, where $C_{\Omega}$ depends on the geometry of $\partial \Omega$. For practical estimates of the latter term, we refer to Dörfler [Dör96, Section 5] and Morin et al. [MNS, inequality (3.3)]. Another practical choice of $\hat{g}$ and $\hat{g}_{h}$ is given in Dörfler and Rumpf [DR98, Section 7].

Proof of Theorem 4.1. 1. We first investigate the relationship between error and residual that is relevant for statement (i). Let $\omega$ be a nonempty subdomain of $\Omega$ and $\varphi \in V$ with $\operatorname{supp} \varphi \subseteq \bar{\omega}$. Using (3.4) and Schwarz inequalities, we see that

$$
\begin{aligned}
\left\langle\mathcal{R}_{h}, \varphi\right\rangle & =\int_{\Omega}\left[a\left(\nabla u_{h}\right)-a(\nabla u)\right] \cdot \nabla \varphi \leq \int_{\omega}\left|N\left(\nabla u_{h}\right)-N(\nabla u)\right||\nabla \varphi| \\
& \leq \sqrt{2} e_{\omega}\left(u_{h}, u\right)\left(\int_{\omega} \frac{|\nabla \varphi|^{2}}{A(\nabla u)+A\left(\nabla u_{h}\right)}\right)^{1 / 2} .
\end{aligned}
$$

Since $u \in W_{\infty}^{1}(\Omega)$ and

$$
\frac{1}{A(\nabla u)+A\left(\nabla u_{h}\right)} \leq \frac{1}{A(\nabla u)}=\Lambda(\nabla u)
$$

we obtain

$$
\left\|\mathcal{R}_{h}\right\|_{-1,2 ; \omega} \leq \sqrt{2} \sup _{\omega} \Lambda(\nabla u)^{1 / 2} e_{\omega}\left(u_{h}, u\right) .
$$

2. To finish the proof of statement (i), we combine inequality (4.5) with the following "lower" bound for the residual:

$$
\eta_{h}(T) \preccurlyeq\left\|\mathcal{R}_{h}\right\|_{-1,2 ; T^{*}}+\|h(\bar{H}-H)\|_{0,2 ; T^{*}} .
$$

This bound is proved by a constructive argument due to Verfürth, which is now quite standard: see e.g., Ver96, Section 1.2]. Let $T \in \mathcal{T}_{h}$ and set $\psi_{T}:=\prod_{z \in \mathcal{N}_{h} \cap T} \phi_{h}^{z} \in$ $P_{d+1}(T)$. Since $|T| \preccurlyeq \int_{T} \psi_{T}$ (see e.g., [Cia78, Exercise 4.1.1]), we obtain

$$
\|\bar{H}\|_{0,2 ; T}^{2} \preccurlyeq \int_{T} \bar{H} \varphi_{T}
$$

for $\varphi_{T}:=\bar{H} \psi_{T} \in P_{d+1}(T)$. Using (3.6) with $\varphi=-\varphi_{T}$ and $\operatorname{supp} \varphi_{T}=T$, we derive

$$
\int_{T} \bar{H} \varphi_{T}=\left\langle\mathcal{R}_{h},-\varphi_{T}\right\rangle+\int_{T}(\bar{H}-H) \varphi_{T} .
$$

We estimate the right-hand side with the help of (see [Ver96, Lemma 3.3])

$$
\left\|\nabla \varphi_{T}\right\|_{0,2 ; T} \preccurlyeq h_{T}^{-1}\left\|\varphi_{T}\right\|_{0,2 ; T} \preccurlyeq h_{T}^{-1}\|\bar{H}\|_{0,2 ; T}
$$

and (4.2), insert the resulting inequality in (4.7), and obtain

$$
\|\bar{H}\|_{0,2 ; T} \preccurlyeq h_{T}^{-1}\left\|\mathcal{R}_{h}\right\|_{-1,2 ; T}+\|\bar{H}-H\|_{0,2 ; T} .
$$


The triangle inequality then yields the following partial version of (4.6):

$$
h_{T}\|H\|_{0,2 ; T} \preccurlyeq\left\|\mathcal{R}_{h}\right\|_{-1,2 ; T}+\|h(\bar{H}-H)\|_{0,2 ; T} .
$$

In a similar manner (use $\varphi_{S}:=\llbracket a\left(\nabla u_{h}\right) \cdot n \rrbracket_{\mid S} \psi_{S}$ with $\psi_{S}:=\prod_{z \in \mathcal{N}_{h} \cap S} \phi_{h}^{z}$ instead of $\varphi_{T}$ for every side $S \in \mathcal{S}_{h}^{\circ}$; cf. [Ver96, (1.26)]), we derive

$$
h_{T}^{1 / 2}\left\|\llbracket a\left(\nabla u_{h}\right) \cdot n \rrbracket\right\|_{0,2 ; \partial T \backslash \partial \Omega} \preccurlyeq\left\|\mathcal{R}_{h}\right\|_{-1,2 ; T^{*}}+\|h H\|_{0,2 ; T^{*}} .
$$

The last two inequalities prove (4.6) and thus statement (i).

3. Next, we investigate the relationship of error and residual that is relevant for statement (ii). Generalizing (3.5), we test (3.4) with $\varphi=\left(u_{h}-\hat{g}_{h}\right)-(u-\hat{g}) \in V$ so as to obtain

$$
e_{\Omega}\left(u_{h}, u\right)^{2}=\left\langle\mathcal{R}_{h},\left(u_{h}-\hat{g}_{h}\right)-(u-\hat{g})\right\rangle+\int_{\Omega}\left[a\left(\nabla u_{h}\right)-a(\nabla u)\right] \cdot \nabla\left(\hat{g}_{h}-\hat{g}\right) .
$$

We continue with the first term on the right-hand side, which is the crucial one:

$$
\left\langle\mathcal{R}_{h},\left(u_{h}-\hat{g}_{h}\right)-(u-\hat{g})\right\rangle \leq\left\|\mathcal{R}_{h}\right\|_{-1,2 ; \Omega}\left(\left\|\nabla\left(u_{h}-u\right)\right\|_{0,2 ; \Omega}+\left\|\nabla\left(\hat{g}_{h}-\hat{g}\right)\right\|_{0,2 ; \Omega}\right) .
$$

Suppose that (4.3) holds. Using $\left|s \nabla u_{h}+(1-s) \nabla u\right| \leq|\nabla u|+M$ for $s \in[0,1]$ in

$$
e_{\Omega}\left(u_{h}, u\right)^{2}=\int_{\Omega} \int_{0}^{1} D^{2} A\left(s \nabla u_{h}+(1-s) \nabla u\right) \nabla\left(u_{h}-u\right) \cdot \nabla\left(u_{h}-u\right) d s,
$$

we get

$$
\inf _{\Omega} \lambda^{M}(\nabla u)\left\|\nabla\left(u_{h}-u\right)\right\|_{0,2 ; \Omega}^{2} \leq e_{\Omega}\left(u_{h}, u\right)^{2} .
$$

This, the preceding inequality for $\left\langle\mathcal{R}_{h},\left(u_{h}-\hat{g}_{h}\right)-(u-\hat{g})\right\rangle$, and the inequality

$$
\forall r, s \geq 0, \epsilon>0 \quad r s \leq \frac{\epsilon}{2} r^{2}+\frac{1}{2 \epsilon} s^{2}
$$

imply

$$
\begin{gathered}
\left\langle\mathcal{R}_{h},\left(u_{h}-\hat{g}_{h}\right)-(u-\hat{g})\right\rangle \leq \frac{1}{4} e_{\Omega}\left(u_{h}, u\right)^{2}+\frac{1}{2} \inf _{\Omega} \lambda^{M}(\nabla u)\left\|\nabla\left(\hat{g}_{h}-\hat{g}\right)\right\|_{0,2 ; \Omega}^{2} \\
+\frac{3}{2}\left[\inf _{\Omega} \lambda^{M}(\nabla u)\right]^{-1}\left\|\mathcal{R}_{h}\right\|_{-1,2 ; \Omega}^{2} .
\end{gathered}
$$

For the second term in the right-hand side of [4.10), we derive (cf. step 1)

$$
\int_{\Omega}\left[a\left(\nabla u_{h}\right)-a(\nabla u)\right] \cdot \nabla\left(\hat{g}_{h}-\hat{g}\right) \leq \frac{1}{4} e_{\Omega}\left(u_{h}, u\right)^{2}+2 \sup _{\Omega} \Lambda(\nabla u)\left\|\nabla\left(\hat{g}_{h}-\hat{g}\right)\right\|_{0,2 ; \Omega}^{2} .
$$

Consequently, (4.10) and $\lambda^{M}(p) \leq \Lambda(p)$ for all $p \in \mathbb{R}^{d}$ yield the inequality

$$
e_{\Omega}\left(u_{h}, u\right) \leq\left(3 \frac{\left\|\mathcal{R}_{h}\right\|_{-1,2 ; \Omega}^{2}}{\inf _{\Omega} \lambda^{M}(\nabla u)}+5 \sup _{\Omega} \Lambda(\nabla u)\left\|\nabla\left(\hat{g}_{h}-\hat{g}\right)\right\|_{0,2 ; \Omega}^{2}\right)^{1 / 2},
$$

provided (4.3) holds.

4. We finish the proof of statement (ii) by showing the upper bound

$$
\left\|\mathcal{R}_{h}\right\|_{-1,2 ; \Omega} \preccurlyeq\left[\sum_{T \in \mathcal{T}_{h}} \eta_{h}(T)^{2}\right]^{1 / 2}
$$


for the residual. This is done along standard lines; see e.g., [Ver96 Section 1.2]. Let $\varphi \in V$, and let $\Pi_{h} \varphi$ be its interpolant from $\$ 3.3$ Using (3.7), (3.3), (3.6), (3.8) with $q=r=2$, and (2.3), we derive

$$
\begin{aligned}
\left\langle\mathcal{R}_{h}, \varphi\right\rangle & =\left\langle\mathcal{R}_{h}, \varphi-\Pi_{h} \varphi\right\rangle \preccurlyeq \sum_{T \in \mathcal{T}_{h}} \eta_{h}(T)\|\nabla \varphi\|_{0,2 ; B_{h}(T)} \\
& \preccurlyeq\left[\sum_{T \in \mathcal{T}_{h}} \eta_{h}(T)^{2}\right]^{1 / 2}\|\nabla \varphi\|_{0,2 ; \Omega},
\end{aligned}
$$

that is, (4.14). Inserting (4.14) into (4.13), we conclude statement (ii).

The following remarks on Theorem 4.1 continue the discussion of the introduction $\$ 1$ and prepare for the second approach.

- Neglecting the oscillation of $H$ and multiplicative constants depending on the shape-regularity $\gamma_{h}$, the local lower bound means that the indicator $\eta_{h}(T)$ underestimates the local error $e_{T^{*}}\left(u_{h}, u\right)$ by a factor greater than

$$
\inf _{T^{*}} \Lambda(\nabla u)^{-1 / 2} \geq 1
$$

- Assuming that $g_{h}=g$ and that the upper bound holds, and again neglecting multiplicative constants depending on $\gamma_{h}$, one expects that, typically, $\eta_{h}(T)$ underestimates the local error by a factor less than

$$
\sup _{T^{*}} \lambda^{M}(\nabla u)^{-1 / 2} \geq 1
$$

- In contrast to linear elliptic problems, there is a (possibly considerable) gap between upper and lower bounds depending on the unknown exact solution $u$.

The afore-mentioned drawbacks of Approach I arise from the way in which we relate the error $e_{\Omega}\left(u_{h}, u\right)$ and the residual $\mathcal{R}_{h}$. Our choice to measure the residual $\mathcal{R}_{h}$ in the dual norm $\|\cdot\|_{-1,2 ; \Omega}$ forces us to use a nonlocal concept of ellipticity of the underlying operator $-\operatorname{div}(a(\nabla \cdot))$ in (4.11). Moreover, relying on the exact solution $u$ entails that the ellipticity and continuity properties linking error and residual are not computable. Finally, Approach I does not really face a main difficulty of the operator $-\operatorname{div}(a(\nabla \cdot))$, namely its nonuniform ellipticity: the assumptions (4.3) and $u \in W_{\infty}^{1}(\Omega)$ yield a situation as for uniform elliptic operators.

\section{Approach II}

We present and analyze the second approach, which constitutes the core of this article. The main result is Theorem 5.1] which contains Result II as a special case.

5.1. Controlling with local ellipticity. The final remarks of 4 indicate that it may be convenient to measure the residual $\mathcal{R}_{h}$ in a weighted way involving the computed solution $u_{h}$. While the suitable way for the lower bound is immediate, the one for the upper bound is more involved. We now turn to it.

As mentioned before, a key issue is the application of inequality (4.11). An alternative for this step arises from the following observation (suppose for a moment $\hat{g}_{h}=\hat{g}=0$ ). The test function in (4.15), which is relevant for the preceding step 3, is $\varphi=u_{h}-u$, and one may already switch to the local error $e_{B_{h}(T)}\left(u_{h}, u\right)$ before the last step in (4.15). This requires a local counterpart of (4.11). But this is only useful if we are able to compute the ellipticity properties (because otherwise we will end 
up with the same local indicators). We thus need an estimate of $\nabla\left(u_{h}-u\right)_{\mid B_{h}(T)}$ in terms of $e_{B_{h}(T)}\left(u_{h}, u\right)$ and some additional computable quantities. Unfortunately, for any $p_{1} \in \mathbb{R}^{d}$, we have (using the notation in Lemma 3.1)

$$
\frac{\left|p_{1}-p_{2}\right|^{2}}{\frac{1}{2}\left|N\left(p_{1}\right)-N\left(p_{2}\right)\right|^{2}\left(A\left(p_{1}\right)+A\left(p_{2}\right)\right)} \longrightarrow \infty \quad \text { as } \quad\left|p_{2}\right| \rightarrow \infty .
$$

Therefore, such an estimate with additional quantities depending solely on $\nabla u_{h}$ does not appear to be possible without further assumptions; see also $\$ 5.4$. Instead of allowing some dependence on $\nabla u$ (which would entail drawbacks as in Approach I), we propose to rely on the following estimate.

Lemma 5.1. Let $p_{1}, p_{2} \in \mathbb{R}^{d}$ and set $P_{i}:=\left(p_{i},-1\right)$ for $i=1,2$. We have

$$
\left|p_{1}-p_{2}\right| \frac{1}{\left|P_{1}\right|^{2}} \leq 2\left|\frac{P_{1}}{\left|P_{1}\right|}-\frac{P_{2}}{\left|P_{2}\right|}\right| \sqrt{\left|P_{1}\right|} \frac{1}{\sqrt{\left|P_{1}\right|}}+\left|\frac{P_{1}}{\left|P_{1}\right|}-\frac{P_{2}}{\left|P_{2}\right|}\right|^{2}\left|P_{2}\right| .
$$

Proof. We first observe that

$$
\left|p_{1}-p_{2}\right|=\left|P_{1}-P_{2}\right| \leq\left|\frac{P_{1}}{\left|P_{1}\right|}-\frac{P_{2}}{\left|P_{2}\right|}\right|\left|P_{1}\right|+|| P_{1}|-| P_{2}|| .
$$

Moreover, we estimate

$$
\begin{aligned}
|| P_{1}|-| P_{2}|| \frac{1}{\left|P_{1}\right|^{2}} & \leq\left|\frac{\left|P_{1}\right|-\left|P_{2}\right|}{\left|P_{1}\right|\left|P_{2}\right|}\right|+\left|\frac{\left|P_{1}\right|-\left|P_{2}\right|}{\left|P_{1}\right|}\left(\frac{1}{\left|P_{1}\right|}-\frac{1}{\left|P_{2}\right|}\right)\right| \\
& \leq\left|\frac{1}{\left|P_{1}\right|}-\frac{1}{\left|P_{2}\right|}\right|+\left|\frac{1}{\left|P_{1}\right|}-\frac{1}{\left|P_{2}\right|}\right|^{2}\left|P_{2}\right| .
\end{aligned}
$$

We insert the last inequality in the first one multiplied by $1 /\left|P_{1}\right|^{2}$, and establish the claim by observing that $\left|P_{1}\right| \geq 1$ and $|1 /| P_{1}|-1 /| P_{2}|| \leq\left|P_{1} /\right| P_{1}\left|-P_{2} /\right| P_{2}||$.

The following fact will be important: the weight of the estimated term $\left|p_{1}-p_{2}\right|$ is $\left|P_{1}\right|^{-2}$ and the weight of the leading order term $\left|P_{1} /\right| P_{1}\left|-P_{2} /\right| P_{2}|| \sqrt{\left|P_{1}\right|}$ on the right-hand side is $\left|P_{1}\right|^{-1 / 2}$, so the squared quotient of these weights is $\left|P_{1}\right|^{-3}=$ $\lambda\left(p_{1}\right)$.

5.2. Weighted indicators. We introduce the indicators for the second approach. Their main building block is quite similar to the indicators (4.1) of the first approach: for any simplex $T \in \mathcal{T}_{h}$, we define $\widetilde{\eta}_{h}(T) \geq 0$ by

$$
\widetilde{\eta}_{h}(T)^{2}=h_{T}\left\|\llbracket a\left(\nabla u_{h}\right) \cdot n \rrbracket\right\|_{0,2 ; \partial T \backslash \partial \Omega}^{2}+h_{T}^{d}\|H\|_{0, d ; T}^{2} .
$$

Note that in the setting of the introduction, that is $d=2$, we have $\eta(T)=\widetilde{\eta}(T)$ for all $T \in \mathcal{T}_{h}$.

Remark 5.1 (Scaling of the interior residual). At first glance, the interior residual $h_{T}^{d}\|H\|_{0, d ; T}^{2}$ seems to be rather unusual. However, we have (recall that $d \geq 2$ )

$$
h_{T}^{2}\|H\|_{0,2 ; T}^{2} \preccurlyeq h_{T}^{d}\|H\|_{0, d ; T}^{2}
$$

thanks to a Hölder inequality; and, if $H$ is piecewise polynomial, in addition,

$$
h_{T}^{d}\|H\|_{0, d ; T}^{2} \preccurlyeq h_{T}^{2}\|H\|_{0,2 ; T}^{2}
$$

thanks to an inverse estimate (see e.g., [Cia78, (3.2.33)]); the hidden constant in the last inequality depends not only on the shape-regularity $\gamma_{h}$ but also on the maximal polynomial degree of $H$. 
Moreover, we need quantities that are used as local weights. Set

$$
\begin{gathered}
\Lambda_{h}(T):=\sup _{T^{*}} \Lambda\left(\nabla u_{h}\right), \quad Q_{h}(T):=\sup _{B_{h}(T)} Q\left(\nabla u_{h}\right), \\
\lambda_{h}(T):=Q_{h}(T)^{-2}\left(\inf _{B_{h}(T)} \sqrt{1+\left|\nabla u_{h}\right|^{2}}\right),
\end{gathered}
$$

for any $T \in \mathcal{T}_{h}$. These quantities are strongly related to the "continuous" quantities $\lambda, \Lambda$ and $Q$ : for example, if $\nabla u_{h}=p$ on $B_{h}(T)$, then $\lambda_{h}(T)=\lambda(p)$.

5.3. Lower and upper bounds. With the help of the computable quantities in the previous subsection, $e_{\Omega}\left(u_{h}, u\right)$ can be controlled in the following way.

Theorem 5.1 (Approach II). Let $u$ be a solution of Problem 2.1, and suppose that $H \in L_{d}(\Omega)$ and (2.2) holds. Moreover, let $u_{h}$ be the solution of Problem 2.2. Then:

(i) (Local lower bounds) For any $T \in \mathcal{T}_{h}$, we have

$$
\frac{\widetilde{\eta}_{h}(T)}{\Lambda_{h}(T)^{1 / 2}} \preccurlyeq e_{T^{*}}\left(u_{h}, u\right)+\frac{\left\|h^{d / 2}(\bar{H}-H)\right\|_{0, d ; T^{*}}}{\Lambda_{h}(T)^{1 / 2}} .
$$

(ii) (Conditional global upper bound) There exists a constant $C$, depending only on the shape-regularity $\gamma_{h}$, such that if

$$
M_{h}:=\max _{T \in \mathcal{T}_{h}} Q_{h}(T) h_{T}^{-d / 2} \widetilde{\eta}_{h}(T) \leq C,
$$

then

$$
e_{\Omega}\left(u_{h}, u\right) \preccurlyeq\left(\sum_{T \in \mathcal{T}_{h}} \frac{\widetilde{\eta}_{h}(T)^{2}}{\lambda_{h}(T)}+\int_{\Omega}\left|\nabla\left(\hat{g}_{h}-\hat{g}\right)\right|^{2} \Lambda\left(\nabla u_{h}\right)\right)^{1 / 2} .
$$

Here, $\hat{g}_{h}$ and $\hat{g}$ are $H^{1}(\Omega)$-extensions of $g_{h}$ and $g$.

Remark 5.2 (On the choice of $\hat{g}$ and $\hat{g}_{h}$ ). Since $\Lambda\left(\nabla u_{h}\right) \leq 1$, we can treat the term $\left(\int_{\Omega}\left|\nabla\left(\hat{g}_{h}-\hat{g}\right)\right|^{2} \Lambda\left(\nabla u_{h}\right)\right)^{1 / 2}$ as in Remark 4.1

Proof of Theorem 5.1. 1. As before, we first investigate the relationship between error and residual that is relevant for statement (i). Proceeding as in step 1 of the proof of Theorem 4.1 but using

$$
\frac{1}{A(\nabla u)+A\left(\nabla u_{h}\right)} \leq \frac{1}{A\left(\nabla u_{h}\right)}
$$

instead of (4.4), we obtain

$$
\left\|\mathcal{R}_{h}\right\|_{-1,2 ; \omega} \leq \sqrt{2} \sup _{\omega} \Lambda\left(\nabla u_{h}\right)^{1 / 2} e_{\omega}\left(u_{h}, u\right) .
$$

2. To conclude the proof of statement (i), we establish the variant

$$
\widetilde{\eta}_{h}(T) \preccurlyeq\left\|\mathcal{R}_{h}\right\|_{-1,2 ; T^{*}}+\left\|h^{d / 2}(\bar{H}-H)\right\|_{0, d ; T^{*}}
$$

of (4.6) by modifying the argument. Since $|T| \preccurlyeq \int_{T} \psi_{T}$, we have

$$
\|\bar{H}\|_{0, d ; T}^{d} \preccurlyeq \int_{T} \bar{H} \varphi_{T}
$$

for $\varphi_{T}:=\bar{H}|\bar{H}|^{d-2} \psi_{T}$. Let $d^{*}:=d /(d-1)$ be the dual exponent of $d$. This time we estimate the right-hand side of formula (4.8) with the help of

$$
\left\|\nabla \varphi_{T}\right\|_{0,2 ; T} \preccurlyeq h_{T}^{-d / 2}\left\|\varphi_{T}\right\|_{0, d^{*} ; T} \preccurlyeq h_{T}^{-d / 2}\|\bar{H}\|_{0, d ; T}^{d-1}
$$


(cf. [Ver96, Lemma 3.3]) and obtain

$$
h_{T}^{d / 2}\|H\|_{0, d ; T} \preccurlyeq\left\|\mathcal{R}_{h}\right\|_{-1,2 ; T}+\left\|h^{d / 2}(\bar{H}-H)\right\|_{0, d ; T} .
$$

Combining this with

$$
h_{T}^{1 / 2}\left\|\llbracket a\left(\nabla u_{h}\right) \cdot n \rrbracket\right\|_{0,2 ; \partial T \backslash \partial \Omega} \preccurlyeq\left\|\mathcal{R}_{h}\right\|_{-1,2 ; T^{*}}+\left\|h^{d / 2} H\right\|_{0, d ; T^{*}},
$$

which follows from (4.9) and (5.3), yields (5.6), and thus statement (i) is proved.

3. In order to prepare the proof of statement (ii), we first establish the counterpart of step 4 in the proof of Theorem 4.1 More precisely, taking into account the discussion of 5.1 we show that

$$
\left\langle\mathcal{R}_{h}, \varphi\right\rangle \preccurlyeq \sum_{T \in \mathcal{T}_{h}} h_{T}^{-d / 2} \widetilde{\eta}_{h}(T)\|\nabla \varphi\|_{0,1 ; B_{h}(T)}
$$

for all $\varphi \in V$. Let $\varphi \in V$, and let $\Pi_{h} \varphi$ be its interpolant from 3.3. Using (3.7), (3.3), and (3.6), we get

$$
\left\langle\mathcal{R}_{h}, \varphi\right\rangle=\sum_{T \in \mathcal{T}_{h}}\left[\frac{1}{2} \int_{\partial T \backslash \partial \Omega} \llbracket a\left(\nabla u_{h}\right) \cdot n \rrbracket\left(\Pi_{h} \varphi-\varphi\right)+\int_{T} H\left(\Pi_{h} \varphi-\varphi\right)\right] .
$$

For the terms involving the prescribed mean curvature $H$, we obtain

$$
\int_{T} H\left(\Pi_{h} \varphi-\varphi\right) \preccurlyeq\|H\|_{0, d ; T}\|\nabla \varphi\|_{0,1 ; B_{h}(T)}
$$

by (3.8a) with $r=d^{*}$ and $q=1$, and for those involving the jump,

$$
\int_{\partial T \backslash \partial \Omega} \llbracket a\left(\nabla u_{h}\right) \cdot n \rrbracket\left(\Pi_{h} \varphi-\varphi\right) \preccurlyeq\left\|\llbracket a\left(\nabla u_{h}\right) \cdot n \rrbracket\right\|_{0, \infty ; \partial T \backslash \partial \Omega}\|\nabla \varphi\|_{0,1 ; B_{h}(T)}
$$

by (3.8b) with $q=1$. We insert the last two inequalities in (5.8) and use

$$
\left\|\llbracket a\left(\nabla u_{h}\right) \cdot n \rrbracket\right\|_{0, \infty ; \partial T \backslash \partial \Omega}+\|H\|_{0, d ; T} \preccurlyeq h_{T}^{-d / 2} \widetilde{\eta}_{h}(T)
$$

to arrive at inequality (5.7).

4. We finish the proof of statement (ii) by establishing the counterpart of step 3 in the proof of Theorem 4.1. Using (4.10) and choosing $\varphi=\left(u_{h}-\hat{g}_{h}\right)-(u-\hat{g}) \in V$ in inequality (5.7), we obtain

$$
\begin{aligned}
e_{\Omega}\left(u_{h}, u\right)^{2} & \leq \int_{\Omega}\left[a\left(\nabla u_{h}\right)-a(\nabla u)\right] \cdot \nabla\left(\hat{g}_{h}-\hat{g}\right) \\
& +C \sum_{T \in \mathcal{T}_{h}} h_{T}^{-d / 2} \widetilde{\eta}_{h}(T)\left\|\nabla\left(\hat{g}_{h}-\hat{g}\right)\right\|_{0,1 ; B_{h}(T)} \\
& +C \sum_{T \in \mathcal{T}_{h}} h_{T}^{-d / 2} \widetilde{\eta}_{h}(T)\left\|\nabla\left(u_{h}-u\right)\right\|_{0,1 ; B_{h}(T)}=: \mathrm{I}+\mathrm{II}+\mathrm{III} .
\end{aligned}
$$

We have to estimate the three terms I, II, III appropriately. Term I is estimated by proceeding similarly to step 1 and using (4.12) with $\epsilon=1 / 3$ :

$$
\begin{aligned}
\mathrm{I} & \leq \sqrt{2} e_{\Omega}\left(u_{h}, u\right)\left(\int_{\Omega}\left|\nabla\left(\hat{g}_{h}-\hat{g}\right)\right|^{2} \Lambda\left(\nabla u_{h}\right)\right)^{1 / 2} \\
& \leq \frac{1}{6} e_{\Omega}\left(u_{h}, u\right)^{2}+3 \int_{\Omega}\left|\nabla\left(\hat{g}_{h}-\hat{g}\right)\right|^{2} \Lambda\left(\nabla u_{h}\right) .
\end{aligned}
$$


Estimating term II with the help of

$$
\begin{aligned}
&\left\|\nabla\left(\hat{g}_{h}-\hat{g}\right)\right\|_{0,1 ; B_{h}(T)} \preccurlyeq h_{T}^{d / 2}\left\|\nabla\left(\hat{g}_{h}-\hat{g}\right)\right\|_{0,2 ; B_{h}(T)} \\
& \preccurlyeq h_{T}^{d / 2}\left(\sup _{B_{h}(T)} \Lambda\left(\nabla u_{h}\right)^{-1 / 2}\right)\left(\int_{B_{h}(T)}\left|\nabla\left(\hat{g}_{h}-\hat{g}\right)\right|^{2} \Lambda\left(\nabla u_{h}\right)\right)^{1 / 2},
\end{aligned}
$$

we obtain again the second term of the right-hand side in (5.10):

$$
\mathrm{II} \preccurlyeq \int_{\Omega}\left|\nabla\left(\hat{g}_{h}-\hat{g}\right)\right|^{2} \Lambda\left(\nabla u_{h}\right)+\sum_{T \in \mathcal{T}_{h}}\left(\sup _{B_{h}(T)} \Lambda\left(\nabla u_{h}\right)^{-1}\right) \widetilde{\eta}_{h}(T)^{2} .
$$

It remains to estimate the crucial term III. Thanks to Lemma 5.1, we have

$$
\begin{aligned}
\left|\nabla\left(u_{h}-u\right)\right| Q_{h}(T)^{-1} \leq 2\left|N\left(\nabla u_{h}\right)-N(\nabla u)\right| & \\
+ & \left|N\left(\nabla u_{h}\right)-N(\nabla u)\right|^{2} A(\nabla u) \quad \text { on } B_{h}(T),
\end{aligned}
$$

whence

$$
\begin{aligned}
\mathrm{III} \leq & C \sum_{T \in \mathcal{T}_{h}} Q_{h}(T) h_{T}^{-d / 2} \widetilde{\eta}_{h}(T) \int_{B_{h}(T)}\left|N\left(\nabla u_{h}\right)-N(\nabla u)\right| \\
& +C \sum_{T \in \mathcal{T}_{h}} Q_{h}(T) h_{T}^{-d / 2} \widetilde{\eta}_{h}(T) \int_{B_{h}(T)}\left|N\left(\nabla u_{h}\right)-N(\nabla u)\right|^{2} A(\nabla u) \\
= & : \mathrm{III}_{a}+\mathrm{III}_{b} .
\end{aligned}
$$

We first consider term $\operatorname{III}_{a}$. The inequality

$$
\int_{B_{h}(T)}\left|N\left(\nabla u_{h}\right)-N(\nabla u)\right| \preccurlyeq h_{T}^{d / 2}\left(\sup _{B_{h}(T)} \Lambda\left(\nabla u_{h}\right)^{1 / 2}\right) e_{B_{h}(T)}\left(u_{h}, u\right)
$$

and the equality $Q_{h}(T)^{2} \sup _{B_{h}(T)} \Lambda\left(\nabla u_{h}\right)=\lambda_{h}(T)^{-1}$ yield

$$
\mathrm{III}_{a} \leq \frac{1}{6} e_{\Omega}\left(u_{h}, u\right)^{2}+C \sum_{T \in \mathcal{T}_{h}} \lambda_{h}(T)^{-1} \widetilde{\eta}_{h}(T)^{2} .
$$

It remains to bound term $\mathrm{III}_{b}$. Let $C$ in (5.4) be the inverse of 6 times the constant hidden in

$$
\mathrm{III}_{b} \preccurlyeq\left(\max _{T \in \mathcal{T}_{h}} Q_{h}(T) h_{T}^{-d / 2} \widetilde{\eta}_{h}(T)\right) e_{\Omega}\left(u_{h}, u\right)^{2} .
$$

Then condition (5.4) implies

$$
\mathrm{III}_{b} \leq \frac{1}{6} e_{\Omega}\left(u_{h}, u\right)^{2} .
$$

To conclude the claimed upper bound for $e_{\Omega}\left(u_{h}, u\right)$, we insert (5.10)-(5.14) in (5.9), and observe that $\sup _{B_{h}(T)} \Lambda\left(\nabla u_{h}\right)^{-1} \leq \lambda_{h}(T)^{-1}$.

We complement the discussion of the introduction and \$4 with the following remarks on Theorem 5.1:

- Neglecting the oscillation of $H$ and multiplicative constants depending on the shape-regularity $\gamma_{h}$, the local lower bound means that the weighted indicator $\Lambda_{h}(T)^{-1 / 2} \widetilde{\eta}_{h}(T)$ does not overestimate the local error $e_{T^{*}}\left(u_{h}, u\right)$.

- Neglecting constants depending on $\gamma_{h}$ and assuming (5.4) and $g_{h}=g$, one expects that, typically, $\lambda_{h}(T)^{-1 / 2} \widetilde{\eta}_{h}(T)$ does not underestimate the local error. 
- The gap between lower and upper bound may be measured locally by the quantity $\Lambda_{h}(T)^{1 / 2} / \lambda_{h}(T)^{1 / 2} \approx Q_{h}(T)^{1 / 2}$.

5.4. On the condition for the upper bound. We discuss some issues that are related to condition (5.4). We first show that (5.1) is relevant, which suggests that the upper bound in Theorem 5.1 cannot hold unconditionally. To this end, let us consider the following example.

Example 5.1 (Part of a catenoid). For $A>1 / \sqrt{2}$, we consider problem (1.1) with data corresponding to the exact solution $u_{A}(x)=-\ln \left(|x|-\sqrt{|x|^{2}-1}\right)$ over the domain $\left.\Omega_{A}=\right] A, A+5\left[^{2}\right.$.

The graph of $u_{A}$ is a part of a minimal surface, namely the catenoid that is centered at the origin of $\mathbb{R}^{3}$ and has the symmetry plane $x_{3}=0$, see e.g., DHKW92, Section 3.5]. The gradient $\nabla u_{A}$ is big near the corner point $(A, A)$, and

$$
\lim _{A \downarrow 1 / \sqrt{2}}\left\|\nabla u_{A}\right\|_{0, \infty ; \Omega_{A}}=\infty .
$$

However, a straightforward computation yields

$$
J\left(u_{A}\right) \leq L
$$

with a constant $L$ not depending on $A$. Let $u_{h, A}$ denote the discrete solution of Problem[2.2] where $g_{h}$ is the interpolant from $\$ 3.3$ of $u_{A \mid \partial \Omega}$. Then, using $|p| \leq A(p)$ for all $p \in \mathbb{R}^{d}, H=0$, and $\left\|\nabla \Pi_{h} u_{A}\right\|_{0,1 ; T} \preccurlyeq\left\|\nabla u_{A}\right\|_{0,1 ; B_{h}(T)}$ for any $T \in \mathcal{T}_{h}$ implies

$$
\left\|\nabla u_{h, A}\right\|_{0,1 ; \Omega_{A}} \leq J\left(u_{h, A}\right) \leq J\left(\Pi_{h} u_{A}\right) \preccurlyeq J\left(u_{A}\right) .
$$

An inverse estimate (see e.g., [Cia78 Theorem 3.2.6]) and (5.16) therefore yield

$$
\left\|\nabla u_{h, A}\right\|_{0, \infty ; \Omega_{A}} \preccurlyeq L h_{\min }^{-1},
$$

where $h_{\min }=\min _{T \in \mathcal{T}_{h}} h_{T}$. In view of (5.15) and (5.17),

$$
\left|\nabla u_{h, A}\right| \ll\left|\nabla u_{A}\right| \quad \text { close to }(A, A),
$$

if $A$ is close to $1 / \sqrt{2}$ and $u_{h, A}$ is a discrete solution over a coarse (i.e., $h_{\min }$ is big) triangulation. Moreover, if $A \downarrow 1 / \sqrt{2}$, the situation considered in 5.1 occurs for $p_{1}=\nabla u_{h, A}(A, A)$ and $p_{2}=\nabla u_{A}(A, A)$.

The condition used in Theorem 4.1 is different from (5.4). So the question arises whether condition (5.4) might be stronger than condition (4.3). However, as can be seen from the following lemma, (4.3) with a small $M$ essentially implies (5.4).

Lemma 5.2. There are constants $M^{*}, H^{*}>0$ depending on the exact solution $u$ and the shape-regularity $\gamma_{h}$ such that condition (4.3) with $M \leq M^{*}$ and

$$
\max _{T \in \mathcal{T}_{h}}\|\bar{H}-H\|_{0, d ; T^{*}} \leq H^{*}
$$

imply condition (5.4).

Proof. Let $T \in \mathcal{T}_{h}$. Similarly to step 2 in the proof of Theorem 5.1 we derive

$$
h_{T}^{-d / 2} \widetilde{\eta}_{h}(T) \preccurlyeq\left\|\mathcal{R}_{h}\right\|_{-1, \infty ; T^{*}}+\|\bar{H}-H\|_{0, d ; T^{*}}
$$

with

$$
\left\|\mathcal{R}_{h}\right\|_{-1, \infty ; T^{*}}:=\sup \left\{\left\langle\mathcal{R}_{h}, \varphi\right\rangle \mid \varphi \in W_{1}^{1}(\Omega), \operatorname{supp} \varphi \subset T^{*},\|\nabla \varphi\|_{0,1 ; T^{*}} \leq 1\right\} .
$$


Equation (3.4) and $\left|a\left(p_{1}\right)-a\left(p_{2}\right)\right| \leq 2 \Lambda\left(p_{1}\right)\left|p_{1}-p_{2}\right|$ for all $p_{1}, p_{2} \in \mathbb{R}^{2}$ imply

$$
\left\|\mathcal{R}_{h}\right\|_{-1, \infty ; T^{*}} \leq 2 \Lambda\left(\inf _{T^{*}}|\nabla u|\right)\left\|\nabla\left(u_{h}-u\right)\right\|_{0, \infty ; \Omega}
$$

Let $M^{*}, H^{*}>0$ (to be chosen in a moment). Using the last two inequalities and supposing that (4.3) with $M \leq M^{*}$ and (5.18) hold, we obtain

$$
\max _{T \in \mathcal{T}_{h}} Q_{h}(T) h_{T}^{-d / 2} \widetilde{\eta}_{h}(T) \preccurlyeq Q\left(\sup _{\Omega}|\nabla u|+M^{*}\right)\left[M^{*} \Lambda\left(\inf _{\Omega}|\nabla u|\right)+H^{*}\right] .
$$

We thus reach our desired conclusion by choosing $M^{*}, H^{*}>0$ so small that the right-hand side times the hidden constant is less than $C$ in (5.4).

We end this section with an interpretation of condition (5.4). The local indicator $h^{-d / 2} \widetilde{\eta}_{h}(T)$ is related to the local residual measured in $W_{\infty}^{-1}(\Omega)$, the dual of $\stackrel{\circ}{W}_{1}^{1}(\Omega)$. The quantity $Q_{h}(T)$ estimates the local $Q(\nabla u)$, which can be understood as quantitative measure for the gap between minimum and maximum eigenvalues of $D^{2} A(\nabla u)$. Thus, condition (5.4) is satisfied, if the local residual is small with respect to the estimated local gap between minimum and maximum eigenvalues.

\section{AdAPTIVITy AND IMPLEMENTATION}

We discuss the application of approaches I and II in an adaptive algorithm. We suppose $g_{h}=g$ for simplicity and focus on the two issues affected by the a posteriori error analysis: stopping test and marking for refinement. In addition, we make some remarks on the implementation that we used for the numerical experiments in $\$ 7$

6.1. Adaptive algorithms. Let us first present and discuss the main steps of an adaptive algorithm. To this end, we replace the subscript $h$ in the preceding sections by an iteration counter $l$.

Algorithm 6.1. Let a tolerance tol $>0$, stopping indicators $\xi_{l}(T), T \in \mathcal{T}_{l}, a$ marking parameter $\theta \in(0,1]$, and marking indicators $\zeta_{l}(T), T \in \mathcal{T}_{l}$, be given.

1. Construct an initial triangulation $\mathcal{T}_{0}$ and set $l:=0$.

2. Solve Problem 2.2 on $\mathcal{T}_{l}$ for $u_{l}$ and compute $\xi_{l}(T)$ for $T \in \mathcal{T}_{l}$.

3. If $\sum_{T \in \mathcal{T}_{l}} \xi_{l}(T)^{2} \leq \mathrm{tol}^{2}$, then $S T O P$.

4. Compute $\zeta_{l}(T)$ for $T \in \mathcal{T}_{l}$.

5. Choose (mark) the smallest subset $\hat{\mathcal{T}}_{l} \subseteq \mathcal{T}_{l}$ with

$$
\sum_{T \in \hat{\mathcal{I}}_{l}} \zeta_{l}(T)^{2} \geq \theta \sum_{T \in \mathcal{T}_{l}} \zeta_{l}(T)^{2} .
$$

6. Refine the simplices in $\hat{\mathcal{T}}_{l}$ to obtain a new triangulation $\mathcal{T}_{l+1}$ in such a way that the shape-regularity is bounded independently of $l$.

7. Increment $l$ and go to 2 .

We refer to this algorithm as Algorithm I, if $\xi_{l}(T)=\zeta_{l}(T)=\eta_{l}(T)$, as Algorithm II, if $\xi_{l}(T)=\lambda_{l}(T)^{-1 / 2} \widetilde{\eta}_{l}(T)$ and $\zeta_{l}(T)=\Lambda_{l}(T)^{-1 / 2} \widetilde{\eta}_{l}(T)$, and as Algorithm IIb, if $\xi_{l}(T)=\zeta_{l}(T)=\lambda_{l}(T)^{-1 / 2} \widetilde{\eta}_{l}(T)$ for all $T \in \mathcal{T}_{l}$.

The stopping test in Step 3 is motivated by the conditional upper bounds of the two approaches in $\S \$ 4$ and 5 However, if Algorithm I has stopped, Theorem 4.1 does not allow us to conclude that $e_{\Omega}\left(u_{l}, u\right) \preccurlyeq$ tol, due to the presence of the noncomputable and problem-dependent term $\inf _{\Omega} \lambda^{M}(\nabla u)$, and because condition (4.3) cannot be verified. If Algorithm II (or IIb) has stopped, then Theorem 5.1 implies $e_{\Omega}\left(u_{l}, u\right) \preccurlyeq$ tol provided (5.4) holds. However, the latter condition cannot 
be verified, as the realistic estimation of $C$ in (5.4) is an open problem. Nevertheless, one could include in Algorithm II the computation of $M_{l}$; its behaviour as $l$ increases may indicate the validity of (5.4) and thus the reliability of the stopping test; see also 7.1

Typically, the iterated application of Step 5 (Dörfler's fixed fraction strategy Dör96]) leads to an almost equidistribution of the marking indicators $\zeta_{l}(T), T \in \mathcal{T}_{l}$. But this does not necessarily imply the desired (see BR78b, Sect. 5]) almost equidistribution of the local errors $e_{T}\left(u_{h}, u\right), T \in \mathcal{T}_{h}$, because $\eta_{l}(T)$ and $\Lambda_{l}(T)^{-1 / 2} \widetilde{\eta}_{l}(T)$ underestimate and $\lambda_{l}(T)^{-1 / 2} \widetilde{\eta}_{l}(T)$ typically overestimates the local error (see $\S \S 4$ and 5 . The performances of these marking indicators are investigated in $\S \S 7.2$ and 7.3 .

6.2. Some implementation issues. We discuss several aspects of our two-dimensional implementations of Algorithms I, II, and IIb.

Problem 2.2 yields a nonlinear discrete system, which is assembled in a standard way. Note that an insufficiently precise approximation of $\int_{\Omega} H \phi_{h}^{z}, z \in \mathcal{N}_{h}^{\circ}$, may produce an unsolvable discrete problem for coarse triangulations; see Proposition 2.1 In our computations, it was sufficient to use a quadrature formula of order 5 for $\int_{T} H \phi_{h}^{z}, z \in \mathcal{N}_{h}^{\circ}, T \in \mathcal{T}_{h}$. We solve the discrete system by using a Newton-like algorithm. The descent direction in each step is computed by means of the conjugate gradient method, which is preconditioned by incomplete Cholesky factorization.

In Step 5 of all three algorithms, we do not actually determine the smallest set. This would require a sort with complexity $\# \mathcal{T}_{l}\left|\log \# \mathcal{T}_{l}\right|$, where $\# \mathcal{T}_{l}$ denotes the number of triangles in $\mathcal{T}_{l}$. Instead we construct an approximation of the smallest set according to Dörfler [Dör96. This construction has complexity $\# \mathcal{T}_{l}$. In all experiments we choose $\theta=0.25$.

Each marked triangle is refined once with the help of the bisection algorithm that is described in Bänsch [Bän91a, Bän91b]. In this way the shape-regularities of refined triangulations depend only on the shape-regularity of the initial triangulation.

Finally, since $\Omega=B(0 ; 1):=\left\{x \in \mathbb{R}^{2}|| x \mid<1\right\}$ in all numerical examples of $\oint 7$ we adapted all three algorithms to this situation. To this end, we modified both approaches in the spirit of Dörfler and Rumpf [DR98] so as to take into account the error due to the approximation of $B(0 ; 1)$; additional terms appear only in the indicators of boundary triangles. Moreover, the coordinates $x=\left(x_{1}, x_{2}\right)$ of new nodes on boundary sides are replaced by $x /|x|$.

\section{NuMERICAL EXPERIMENTS}

Our numerical investigations address the following issues of the algorithms in $\$ 6$.

- The behavior of $M_{l}$ in condition (5.4) as the number $l$ of iterations in Algorithm II increases.

- A comparison of using $\Lambda_{l}(T)^{-1 / 2} \widetilde{\eta}_{l}(T)$ or $\lambda_{l}(T)^{-1 / 2} \widetilde{\eta}_{l}(T)$ as marking indicators $\zeta_{l}(T)$ in Step 5 of Algorithm II or IIb, respectively.

- A comparison of the performances of Algorithms I and II.

7.1. The behavior of $M_{l}$. We study the behavior of $M_{l}$ for the following example:

Example 7.1 (Boundary layer). Let $B>0$ and consider problem (1.1) with data that corresponds to the exact solution $u_{B}(x):=\arctan \left[B\left(|x|^{2}-\frac{1}{4}\right)\right]$ on the domain $\Omega:=\left\{y \in \mathbb{R}^{2}|| y \mid<1\right\}$. 


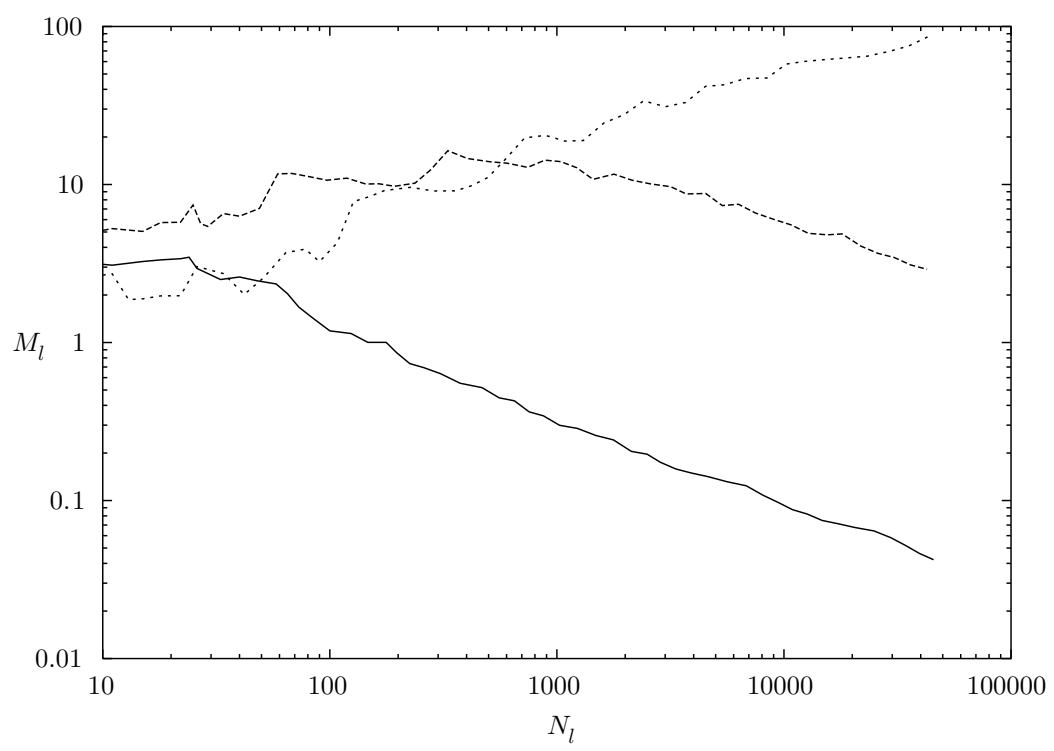

Figure 1. Example 7.1 the behavior of $M_{l}$ versus $N_{l}$ as $l$ increases for $B=1$ (solid), 10 (dashed), 100 (dotted).

The radial symmetric function $u_{B}$ is close to -1 at 0 and close to 1 on the boundary $\partial \Omega$. The transition from -1 to 1 takes place near the circle $\left\{y \in \mathbb{R}^{2} \mid\right.$ $\left.|y|=\frac{1}{2}\right\}$ and gets sharper as $B$ increases: $\lim _{B \uparrow \infty}\left\|\nabla u_{B}\right\|_{0, \infty ; \Omega}=\infty$.

We are interested in the relationship of $M_{l}$ and the number $N_{l}$ of unknows, which measures the size of the discrete Problem 2.2 and increases with $l$. To this end, we execute the loop in Algorithm II as long as $N_{l} \leq 50000$. In Figure 1 the possible behavior of $M_{l}$ is illustrated by three parameter values: for $B=1$, the quantity $M_{l}$ decreases from the start; for $B=10$, it decreases after some increasing; finally, for $B=100$, no decreasing takes place up to $N_{l} \approx 50000$.

This behavior is consistent with the discussion in $\$ 5.4$ if the exact solution is sufficiently regular, refining will reduce the residual measured in $W_{\infty}^{-1}(\Omega)$ and thus the indicators $h_{T}^{-d / 2} \widetilde{\eta}_{l}(T), T \in \mathcal{T}_{l}$, but $\left\|\nabla u_{l}\right\|_{0, \infty ; \Omega}$ and thus the weights $Q_{l}(T)$, $T \in \mathcal{T}_{l}$, may increase for a certain number of iterations; compare with Example 5.1

These numerical results and their discussion indicate:

- Condition (5.4) is asymptotically (i.e., for sufficiently large $l$ ) satisfied, if the exact solution is in $W_{\infty}^{1}(\Omega)$.

- If the exact solution has a steep gradient somewhere, small values of $M_{l}$, and thus the achievement of (5.4), may require discrete problems with (too) many unknowns.

In view of the first item, one expects that the stopping test in Algorithm II is reliable for sufficiently small tolerances tol. We stress that monitoring the computable quantity $M_{l}$ can indicate whether or not (5.4) holds for a reachable number of unknows.

7.2. Local indicators, local error, and marking. It is instructive to compare the relationship between the local error $e_{T}\left(u_{l}, u\right)$ and the indicators $\Lambda_{l}(T)^{-1 / 2} \widetilde{\eta}_{l}(T)$ 


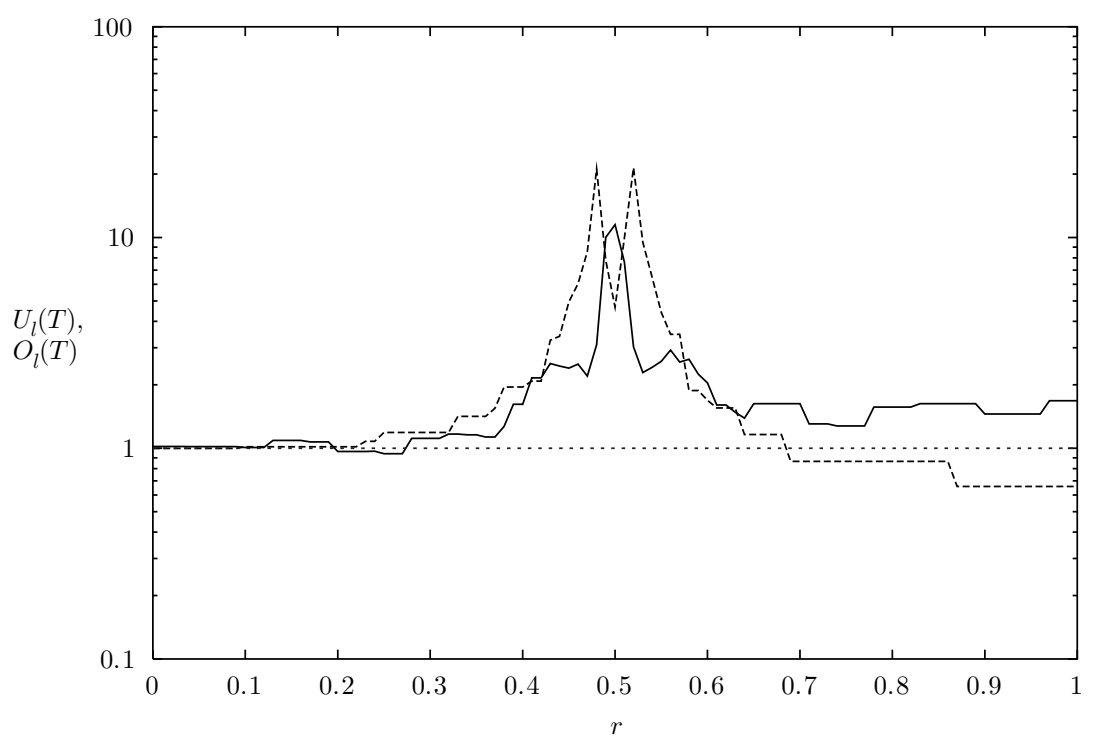

Figure 2. Example $7.1(B=100)$ : local underestimation $U_{l}(T)$ (solid) by $\frac{1}{9} \Lambda_{l}(T)^{-1 / 2} \widetilde{\eta}_{l}(T)$ and overestimation $O_{l}(T)$ (dashed) by $\frac{1}{9} \lambda_{l}(T)^{-1 / 2} \widetilde{\eta}_{l}(T)$.

and $\lambda_{l}(T)^{-1 / 2} \widetilde{\eta}_{l}(T)$. We investigate these relationships for Example 7.1 and the following one.

Example 7.2 (Segment of $R$-sphere). Let $R>1$ and consider problem (1.1) with data that corresponds to the exact solution $u_{R}(x)=\sqrt{R^{2}-|x|^{2}}$ on the domain $\Omega=\left\{y \in \mathbb{R}^{2}|| y \mid<1\right\}$.

The graph of the function $u_{R}$ is an upper segment of the sphere with radius $R>1$ and center at the origin of $\mathbb{R}^{3}$. The modulus $\left|\nabla u_{R}\right|$ of the gradient increases as $|x|$ tends to 1 . Moreover, $\lim _{R \downarrow 1}\left\|\nabla u_{R}\right\|_{\infty ; \Omega}=\infty$.

For the investigation of the local indicators and local error, we define the local under- and overestimation indexes

$$
U_{l}(T):=\frac{e_{T}\left(u_{l}, u\right)}{\frac{1}{9} \Lambda_{l}(T)^{-1 / 2} \widetilde{\eta}_{l}(T)} \quad \text { and } \quad O_{l}(T):=\frac{\frac{1}{9} \lambda_{l}(T)^{-1 / 2} \widetilde{\eta}_{l}(T)}{e_{T}\left(u_{l}, u\right)}
$$

for any $T \in \mathcal{T}_{l}$; the fraction $\frac{1}{9}$ guarantees that $U_{l}(T) \geq 1$ and $O_{l}(T) \geq 1$ for "nonboundary" triangles. The behavior of these indexes along the straight line from $(0,0)$ to $(1,1) / \sqrt{2}$ are depicted in Figure 2 for a discrete solution to Example 7.1 with $B=100$. We observe that $\frac{1}{9} \Lambda_{l}(T)^{-1 / 2} \widetilde{\eta}_{l}(T)$ underestimates and $\frac{1}{9} \lambda_{l}(T)^{-1 / 2} \widetilde{\eta}_{l}(T)$ overestimates the error $e_{T}\left(u_{l}, u\right)$ in regions where $\left|\nabla u_{l}\right|($ and $|\nabla u|)$ is big (we disregard boundary triangles, since they incorporate an additional term in the indicator). Quantitatively, the local overestimation of $\frac{1}{9} \lambda_{l}(T)^{-1 / 2} \widetilde{\eta}_{l}(T)$ is bigger than the local underestimation of $\frac{1}{9} \Lambda_{l}(T)^{-1 / 2} \widetilde{\eta}_{l}(T)$ in Figure 2 Corresponding curves for a discrete solution to Example 7.2 with $R=1+10^{-5}$ are depicted in Figure 3 Here, the local overestimation of $\frac{1}{9} \lambda_{l}(T)^{-1 / 2} \widetilde{\eta}_{l}(T)$ is smaller than the local underestimation of $\frac{1}{9} \Lambda_{l}(T)^{-1 / 2} \widetilde{\eta}_{l}(T)$. 


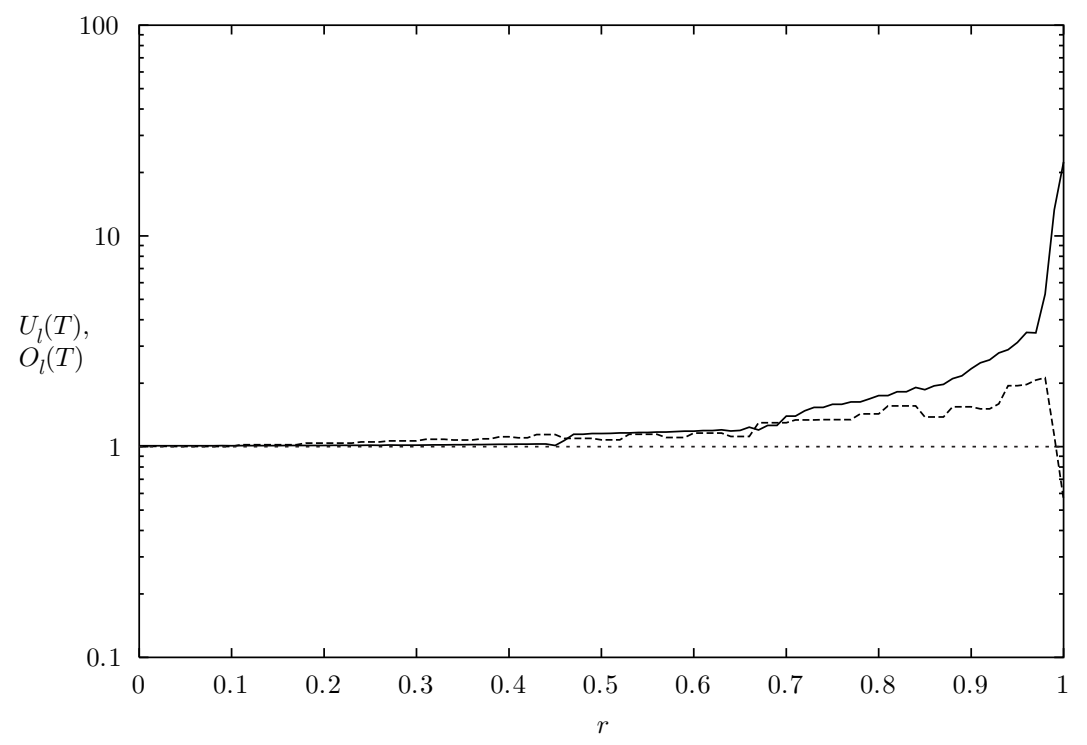

Figure 3. Example $7.2\left(R=1+10^{-5}\right)$ : local underestimation $U_{l}(T)$ (solid) by $\frac{1}{9} \Lambda_{l}(T)^{-1 / 2} \widetilde{\eta}_{l}(T)$ and overestimation $O_{l}(T)$ (dashed) by $\frac{1}{9} \lambda_{l}(T)^{-1 / 2} \widetilde{\eta}_{l}(T)$.

These numerical results and definitions (7.1) indicate the following:

- The constants in Theorem 5.1 which are hidden in $\preccurlyeq$ are of moderate size on meshes with reasonable shape-regularity.

- Typically, the local error $e_{T}\left(u_{l}, u\right)$ is in the interval from $\Lambda_{l}(T)^{-1 / 2} \widetilde{\eta}_{l}(T)$ to $\lambda_{l}(T)^{-1 / 2} \widetilde{\eta}_{l}(T)$ (up to multiplicative constants depending on the initial shape-regularity).

The second item is in accordance with the discussion of $\$ 5.3$ Moreover, it suggests that the estimates in Theorem 5.1 are sharp in the sense that they constitute possible worst cases corresponding to certain directions of the unknown error.

We conclude this subsection with a comparison of Algorithms II and IIb. We applied both algorithms to Examples 7.1] and 7.2 The relationships between global error $e_{\Omega}\left(u_{l}, u\right)$ and number of unknows $N_{l}$ are depicted in Figures 4 and 5, respectively. We observe that Algorithm II performs better for Example 7.1, while Algorithm IIb performs better for Example 7.2 Moreover, the following expectation is confirmed by comparing with Figures 2 and 3 the approach whose marking indicators are closer to the local errors performs better.

Finally, notice the slowdown in the convergence of $e_{\Omega}\left(u_{l}, u\right)$ for Algorithm IIb in Figure 4 for Example 7.1 (which is different from the "waves" in Figure 5 due to the domain approximation). In view of the discussion of Example 5.1 and Figure 2 this may be explained as follows: as long as $\left|\nabla u_{l}\right|$ increases, the typical overestimation of $\lambda_{l}(T)^{-1 / 2} \widetilde{\eta}_{l}(T)$ and thereby the deviation of the local errors from equidistribution becomes more severe. Algorithm II does not exhibit such a slowdown: typically, as long as $\left|\nabla u_{l}\right|$ increases, the underestimation of $\Lambda_{l}(T)^{-1 / 2} \widetilde{\eta}_{l}(T)$ becomes less severe.

We summarize:

- Depending on the example, Algorithm II or IIb performs better. 


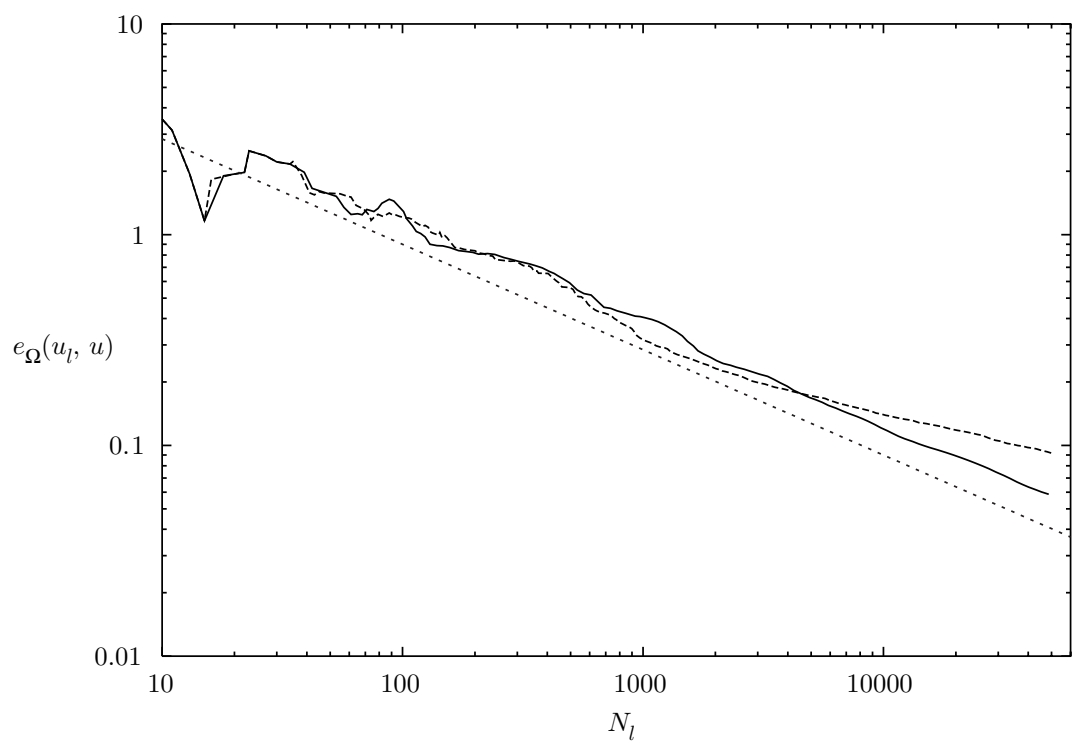

Figure 4. Example 7.1 $(B=100): e_{\Omega}\left(u_{l}, u\right)$ versus $N_{l}$ for Algorithms II (solid) and IIb (dashed). The optimal decay is indicated by the dotted line with slope $-1 / 2$.

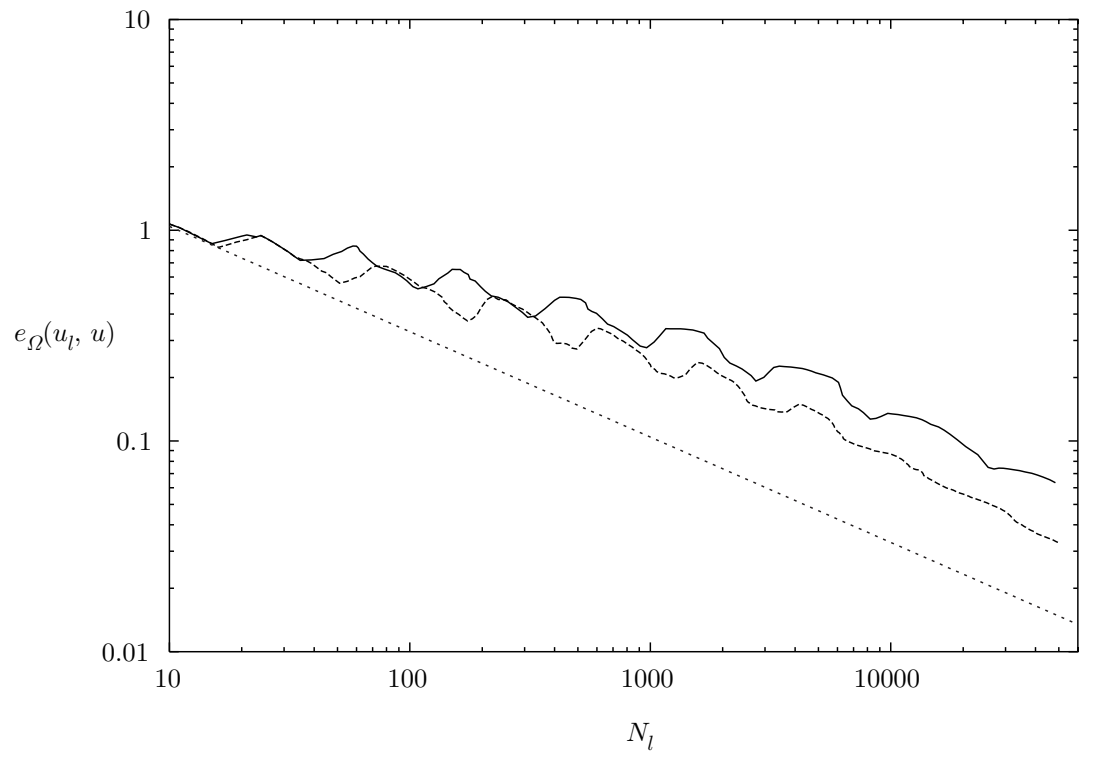

Figure 5. Example $7.2\left(R=1+10^{-5}\right): e_{\Omega}\left(u_{l}, u\right)$ versus $N_{l}$ for Algorithms II (solid) and IIb (dashed). The optimal decay is indicated by the dotted line with slope $-1 / 2$. 


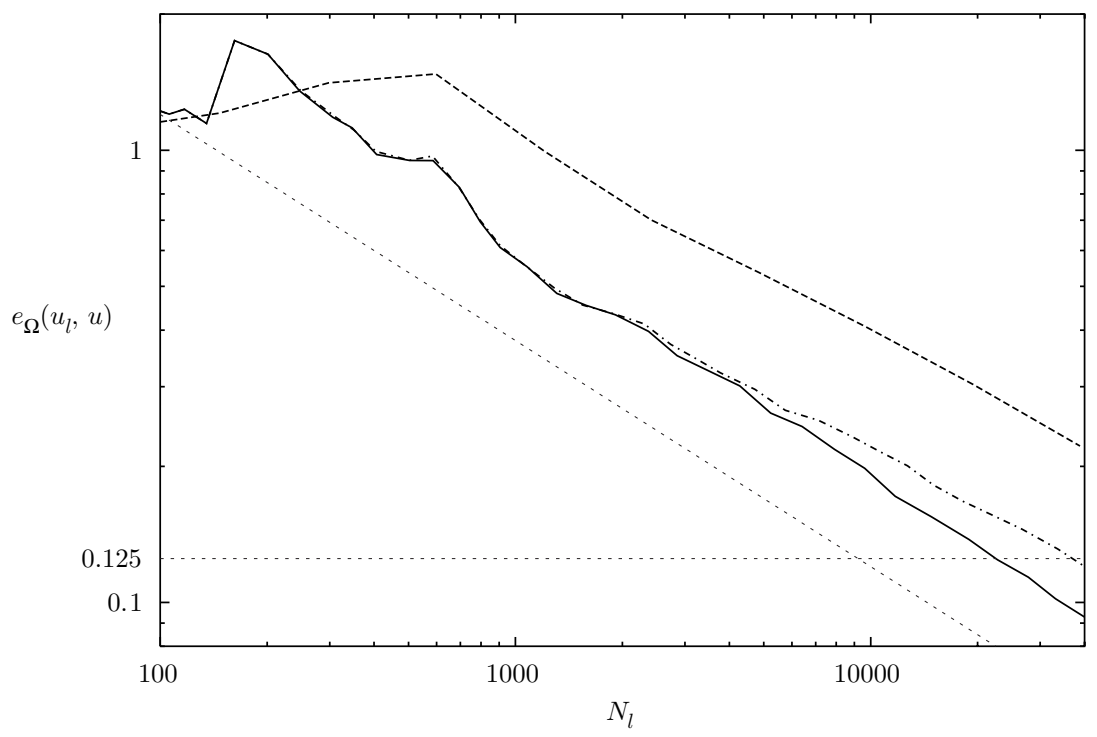

Figure 6. Example $7.1(B=500)$ : $e_{\Omega}\left(u_{l}, u\right)$ versus $N_{l}$ for Algorithms I (dashed-dotted), II (solid) and uniform refinement (dashed). The optimal decay and the value 0.125 are indicated by dotted lines with slope $-1 / 2$ and 0 , respectively.

- In contrast to Algorithm II, Algorithm IIb has the potential risk of a (probably transitory) convergence slowdown.

We prefer Algorithm II to IIb due to the second item and the considerations in $\$ 7.3$ below.

7.3. Algorithm I versus Algorithm II. We finally compare Algorithm I based on approach I with Algorithm II based on approach II.

Inequality (5.3) and $\Lambda_{l}^{-1 / 2}(T) \geq 1$ imply $\eta_{l}(T) \preccurlyeq \Lambda_{l}(T)^{-1 / 2} \widetilde{\eta}_{l}(T)$ for all $T \in \mathcal{T}_{l}$, so $\eta_{l}(T)$ always underestimates the local error more strongly than $\Lambda_{l}(T)^{-1 / 2} \widetilde{\eta}_{l}(T)$. One thus expects that Algorithm II performs better than Algorithm I, especially if $\Lambda(\nabla u)$, i.e., $|\nabla u|$, is a strongly varying function. In fact, for Example 7.1 with $B=500$, Algorithm I needs 39683 unknowns to get $e_{\Omega}\left(u_{h}, u\right) \leq 0.125$, while Algorithm II needs only 22752 for the same goal. See also Figure 6, where the behavior of $e_{\Omega}\left(u_{l}, u\right)$ versus $N_{l}$ is depicted for Algorithms I and II.

Also the case of uniform refinement is shown in Figure 6 as expected, both adaptive algorithms perform better than nonadaptive uniform refinement.

Acknowledgments. The authors wish to thank Professor G. Dziuk for his helpful support, and the referees for their useful comments.

\section{REFERENCES}

[AO00] Mark Ainsworth and J. Tinsley Oden, A posteriori error estimation in finite element analysis, John Wiley, New York, 2000.

[Bän91a] Eberhard Bänsch, Local mesh refinement in 2 and 3 dimensions, IMPACT Comput. Sci. Eng. 3 (1991), no. 3, 181-191. MR 92h:65150 
[Bän91b] Eberhard Bänsch, An adaptive finite-element strategy for the three-dimensional timedependent Navier-Stokes equations, J. Comp. Appl. Math. 36 (1991), no. 1, 3-28. MR 92f:76066

[BR78a] Ivo Babuška and Werner C. Rheinboldt, A-posteriori error estimates for the finite element method, Internat. J. Numer. Methods Engrg. 12 (1978), 1597-1615.

[BR78b] Ivo Babuška and Werner C. Rheinboldt, Error estimates for adaptive finite element computations, SIAM J. Numer. Anal. 15 (1978), 736-754. MR 58:3400

[Cia78] Philippe G. Ciarlet, The finite element method for elliptic problems, Studies in Mathematics and its Applications, vol. 4, North-Holland, Amsterdam, 1978. MR 58:25001

[Cia88] Philippe G. Ciarlet, Introduction to numerical linear algebra and optimization, Cambridge Texts in Applied Mathematics, Cambridge University Press, Cambridge etc., 1989. MR 90k:65001

[DHKW92] Ulrich Dierkes, Stefan Hildebrandt, Albrecht Küster, and Ortwin Wohlrab, Minimal surfaces I. Boundary value problems, Grundlehren der mathematischen Wissenschaften, vol. 295, Springer-Verlag Berlin Heidelberg, 1992. MR 94c:49001a

[Dör96] Willy Dörfler, A convergent adaptive algorithm for Poisson's equation, SIAM J. Numer. Anal. 33 (1996), 1106-1124. MR 97e:65139

[DR98] Willy Dörfler and Martin Rumpf, An adaptive strategy for elliptic problems including a posteriori controlled boundary approximation, Math. Comp. 67 (1998), no. 224, 1361-1382. MR 99b:65141

[Fie98] Francesca Fierro, Numerical approximation of the mean curvature flow with nucleation using implicit time-stepping: an adaptive algorithm, Calcolo 35 (1998), 205224. MR 2001e: 65120

[Gia74] Mariano Giaquinta, On the Dirichlet problem for surfaces of prescribed mean curvature, Manuscripta Math. 12 (1974), 73-86. MR 49:1306

[GT83] David Gilbarg and Neil S. Trudinger, Elliptic partial differential equations of second order, second ed., Springer, New York, 1983. MR 86c:35035

[Hac92] Wolfgang Hackbusch, Elliptic differential equations. Theory and numerical treatment, Springer Series in Computational Mathematics, vol. 18, Springer, New York, 1992. MR 94b:35001

[MNS] Pedro Morin, Ricardo H. Nochetto, and Kunibert G. Siebert, Local problems on stars: a posteriori error estimators, convergence, and performance, Math. Comp., posted on November 7, 2002, PII S0025-5718(02)-01463-1 (to appear in print).

[MNS00] Pedro Morin, Ricardo H. Nochetto, and Kunibert G. Siebert, Data oscillation and convergence of adaptive FEM, SIAM J. Numer. Anal. 38 (2000), no. 2, 466-488. MR 2001g:65157

[Ser69] James Serrin, The problem of Dirichlet for quasilinear elliptic differential equations with many independent variables, Philos. Trans. Roy. Soc. London Ser. A 264 (1969), 413-496. MR 43:7772

[SZ90] L. Ridgway Scott and Shangyou Zhang, Finite element interpolation of nonsmooth functions satisfying boundary conditions, Math. Comp. 54 (1990), no. 190, 483-493. MR 90j:65021

[Ver94] Rüdiger Verfürth, A posteriori error estimates for nonlinear problems. Finite element discretizations of elliptic equations, Math. Comp. 62 (1994), no. 206, 445-475. MR 94j:65136

[Ver96] Rüdiger Verfürth, A review of a posteriori error estimation and adaptive meshrefinement techniques, Advances in Numerical Mathematics, John Wiley, Chichester, 1996.

Dipartimento di Matematica, Università degli Studi di Milano, Via C. Saldini 50, 20133 Milano, Italy

E-mail address: fierro@mat.unimi.it

Institut Für Angewandte Mathematik, Albert-Ludwigs-Universität Freiburg, Hermann-Herder-Str. 10, 79104 Freiburg i. Br., Germany

E-mail address: andy@mathematik.uni-freiburg.de 